\title{
Beyond the spectral standard model: emergence of Pati-Salam unification
}

\author{
Ali H. Chamseddine, ${ }^{a, b}$ Alain Connes ${ }^{b, c, d}$ and Walter D. van Suijlekom ${ }^{e}$ \\ ${ }^{a}$ Physics Department, American University of Beirut, \\ Beirut, Lebanon \\ ${ }^{b}$ I.H.E.S., \\ F-91440 Bures-sur-Yvette, France \\ ${ }^{c}$ College de France, \\ 3 rue Ulm, Fr5005, Paris, France \\ ${ }^{d}$ Department of Mathematics, The Ohio State University, \\ Columbus OH 43210 U.S.A. \\ ${ }^{e}$ Institute for Mathematics, Astrophysics and Particle Physics, Radboud University Nijmegen, \\ Heyendaalseweg 135, 6525 AJ Nijmegen, The Netherlands \\ E-mail: chams@aub.edu.lb, alain@connes.org, waltervs@math.ru.nl
}

ABSTRACT: The assumption that space-time is a noncommutative space formed as a product of a continuous four dimensional manifold times a finite space predicts, almost uniquely, the Standard Model with all its fermions, gauge fields, Higgs field and their representations. A strong restriction on the noncommutative space results from the first order condition which came from the requirement that the Dirac operator is a differential operator of order one. Without this restriction, invariance under inner automorphisms requires the inner fluctuations of the Dirac operator to contain a quadratic piece expressed in terms of the linear part. We apply the classification of product noncommutative spaces without the first order condition and show that this leads immediately to a Pati-Salam $\mathrm{SU}(2)_{R} \times \mathrm{SU}(2)_{L} \times \mathrm{SU}(4)$ type model which unifies leptons and quarks in four colors. Besides the gauge fields, there are 16 fermions in the $(2,2,4)$ representation, fundamental Higgs fields in the $(2,2,1)$, $(2,1,4)$ and $(1,1,1+15)$ representations. Depending on the precise form of the initial Dirac operator there are additional Higgs fields which are either composite depending on the fundamental Higgs fields listed above, or are fundamental themselves. These additional Higgs fields break spontaneously the Pati-Salam symmetries at high energies to those of the Standard Model.

KEywords: Non-Commutative Geometry, Models of Quantum Gravity, Space-Time Symmetries

ARXIV EPRINT: 1304.8050 


\section{Contents}

1 Introduction 1

2 First-order condition and inner fluctuations 3

3 Classification of finite geometries without first order condition 5

$\begin{array}{lll}4 & \text { Summary of tensor notation } & 7\end{array}$

5 Dirac operator and inner fluctuations on $\mathbb{H}_{R} \oplus \mathbb{H}_{L} \oplus M_{4}(\mathbb{C}) \quad 10$

$6 \quad$ The spectral action for the SU $(2)_{R} \times \mathrm{SU}(2)_{L} \times \mathrm{SU}(4)$ model 12

$\begin{array}{lll}7 & \text { Truncation to the standard model } & 15\end{array}$

$\begin{array}{lll}8 & \text { The potential and symmetry breaking } & 17\end{array}$

$\begin{array}{ll}\text { A Detailed calculations for the practitioner } & 22\end{array}$

\section{Introduction}

Noncommutative geometry was shown to provide a promising framework for unification of all fundamental interactions including gravity $[3,5,6,10,12]$. Historically, the search to identify the structure of the noncommutative space followed the bottom-up approach where the known spectrum of the fermionic particles was used to determine the geometric data that defines the space. This bottom-up approach involved an interesting interplay with experiments. While at first the experimental evidence of neutrino oscillations contradicted the first attempt [6], it was realized several years later in 2006 ([12]) that the obstruction to get neutrino oscillations was naturally eliminated by dropping the equality between the metric dimension of space-time (which is equal to 4 as far as we know) and its $K O$ dimension which is only defined modulo 8 . When the latter is set equal to 2 modulo 8 [2, 4] (using the freedom to adjust the geometry of the finite space encoding the fine structure of space-time) everything works fine, the neutrino oscillations are there as well as the see-saw mechanism which appears for free as an unexpected bonus. Incidentally, this also solved the fermionic doubling problem by allowing a simultaneous Weyl-Majorana condition on the fermions to halve the degrees of freedom.

The second interplay with experiments occurred a bit later when it became clear that the mass of the Brout-Englert-Higgs boson would not comply with the restriction (that $m_{H} \succeq 170 \mathrm{Gev}$ ) imposed by the validity of the Standard Model up to the unification scale. This obstruction to lower $m_{H}$ was overcome in [11] simply by taking into account a scalar 
field which was already present in the full model which we had computed previously in [10]. One lesson which we learned on that occasion is that we have to take all the fields of the noncommutative spectral model seriously, without making assumptions not backed up by valid analysis, especially because of the almost uniqueness of the Standard Model (SM) in the noncommutative setting.

The SM continues to conform to all experimental data. The question remains whether this model will continue to hold at much higher energies, or whether there is a unified theory whose low-energy limit is the SM. One indication that there must be a new higher scale that effects the low energy sector is the small mass of the neutrinos which is explained through the see-saw mechanism with a Majorana mass of at least of the order of $10^{11} \mathrm{Gev}$. In addition and as noted above, a scalar field which acquires a vev generating that mass scale can stabilize the Higgs coupling and prevent it from becoming negative at higher energies and thus make it consistent with the low Higgs mass of $126 \mathrm{Gev}$ [11]. Another indication of the need to modify the SM at high energies is the failure (by few percent) of the three gauge couplings to be unified at some high scale which indicates that it may be necessary to add other matter couplings to change the slopes of the running of the RG equations.

This leads us to address the issue of the breaking from the natural algebra $\mathcal{A}$ which results from the classification of irreducible finite geometries of $\mathrm{KO}$-dimension 6 (modulo 8) performed in [9], to the algebra corresponding to the SM. This breaking was effected in $[8,9]$ using the requirement of the first order condition on the Dirac operator. The first order condition is the requirement that the Dirac operator is a derivation of the algebra $\mathcal{A}$ into the commutant of $\hat{\mathcal{A}}=J \mathcal{A} J^{-1}$ where $J$ is the charge conjugation operator. This in turn guarantees the gauge invariance and linearity of the inner fluctuations [7] under the action of the gauge group given by the unitaries $U=u J u J^{-1}$ for any unitary $u \in \mathcal{A}$. This condition was used as a mathematical requirement to select the maximal subalgebra

$$
\mathbb{C} \oplus \mathbb{H} \oplus M_{3}(\mathbb{C}) \subset \mathbb{H}_{R} \oplus \mathbb{H}_{L} \oplus M_{4}(\mathbb{C})
$$

which is compatible with the first order condition and is the main reason behind the unique selection of the SM.

The existence of examples of noncommutative spaces where the first order condition is not satisfied such as quantum groups and quantum spheres provides a motive to remove this condition from the classification of noncommutative spaces compatible with unification [1417]. This study was undertaken in a companion paper [13] where it was shown that in the general case the inner fluctuations of $D$ form a semigroup in the product algebra $\mathcal{A} \otimes \mathcal{A}^{\text {op }}$, and acquire a quadratic part in addition to the linear part. Physically, this new phenomena will have an impact on the structure of the Higgs fields which are the components of the connection along discrete directions. This paper is devoted to the construction of the physical model that describes the physics beyond the Standard Model. The methods used build on previous results and derivations developed over the years. To make this work more accessible we shall attempt to make the paper self-contained by including the parts needed from previous works in a brief form. 
The plan of this paper is as follows. In section 2 we review the effect of removing the first order condition on the form of the inner fluctuations, emphasizing the semigroup structure. In section 3 we modify the classification of irreducible finite geometries in the absence of the first order condition and show that the resultant algebra is, almost uniquely, given by $\mathbb{H}_{R} \oplus \mathbb{H}_{L} \oplus M_{4}(\mathbb{C})$. The model is then based on a noncommutative geometric space formed as a product of a continuous four dimensional space times the above discrete space. The associated connection can be viewed either as a $384 \times 384$ matrices, or in more manageable form as the tensor product of matrices. To present the computations in a comprehensible form that could be checked by others, we give in section 4 a brief review of the tenorial notation we developped before. We stress that all calculations performed in this article using the tensorial method are done by hand, but have the advantage that they could also be checked using algebraic manipulation programs such as Mathematica or Maple. In section 5 we compute the inner fluctuations of the Dirac operator on the above algebra and determine the field content. In section 6 we evaluate the spectral action using a cutoff function and the heat kernel expansion method, where we show that the resultant model is the Pati-Salam [21] $\mathrm{SU}(2)_{R} \times \mathrm{SU}(2)_{L} \times \mathrm{SU}$ (4) type model with all the appropriate Higgs fields necessary to break the symmetry to $U(1)_{\mathrm{em}} \times \mathrm{SU}(3)_{\mathrm{c}}$. In section 7 we show that this model truncates correctly to the SM. In section 8 we analyze the potential and possible symmetry breaking, noting in particular the novel feature that for certain initial configurations of the Dirac operator some of the inner fluctuations represented as Higgs fields are fundamental while others are made of quadratic products of the fundamental ones. For generic initial Dirac operators all Higgs fields are fundamental. Section A is the appendix where all details of the calculation are given and where we illustrate the power and precision of noncommutative geometric methods by showing how all the physical fields arise. This is done to the benefit of researchers interested in becoming practitioners in the field.

\section{First-order condition and inner fluctuations}

We briefly summarize the generalization of inner fluctuations to real spectral triples that fail on the first-order condition, as presented in [13]. In this case, the usual prescription [3] does not apply, since the operator $D+A \pm J A J^{-1}$ with gauge potential $A=\sum_{j} a_{j}\left[D, b_{j}\right]\left(a_{j}, b_{j} \in\right.$ $\mathcal{A})$ does not behave well with respect to the action of the gauge group $\mathcal{U}(\mathcal{A})$. In fact, one would require that conjugation of the fluctuated Dirac operator by the unitary operator $U:=u J u J^{-1}$ for $u \in \mathcal{U}(\mathcal{A})$ can be implemented by a usual type of gauge transformation $A \mapsto A^{u}=u\left[D, u^{*}\right]+u A u^{*}$ so that

$$
D+A \pm J A J^{-1} \mapsto U\left(D+A \pm J A J^{-1}\right) U^{*} \equiv D+A^{u} \pm J A^{u} J^{-1}
$$

However, the simple argument only works if $\left[\mathrm{JuJ}^{-1}, A\right]=0$ for gauge potentials $A$ of the above form and $u \in \mathcal{U}(\mathcal{A})$, that is, if the first-order condition is satisfied.

For real spectral triples that possibly fail on the first-order condition one starts with a self-adjoint, universal one-form

$$
A=\sum_{j} a_{j} \delta\left(b_{j}\right) ; \quad\left(a_{j}, b_{j} \in \mathcal{A}\right)
$$


The inner fluctuations of a real spectral triple $(\mathcal{A}, \mathcal{H}, D ; J)$ are then given by

$$
D^{\prime}=D+A_{(1)}+\tilde{A}_{(1)}+A_{(2)}
$$

where

$$
\begin{aligned}
& A_{(1)}:=\sum_{j} a_{j}\left[D, b_{j}\right], \\
& \tilde{A}_{(1)}:=\sum_{j} \hat{a}_{j}\left[D, \hat{b}_{j}\right] ; \quad \hat{a}_{i}=J a_{i} J^{-1}, \quad \hat{b}_{i}=J b_{i} J^{-1}, \\
& A_{(2)}:=\sum_{j} \hat{a}_{j}\left[A_{(1)}, \hat{b}_{j}\right]=\sum_{j, k} \hat{a}_{j} a_{k}\left[\left[D, b_{k}\right], \hat{b}_{j}\right] .
\end{aligned}
$$

Clearly $A_{(2)}$ which depends quadratically on the fields in $A_{(1)}$ vanishes when the first order condition is satisfied, thus reducing to the usual formulation of inner fluctuations. As such, we will interpret the terms $A_{(2)}$ as non-linear corrections to the first-order, linear inner fluctuations $A_{(1)}$ of $(\mathcal{A}, \mathcal{H}, D ; J)$.

The need for such quadratic terms can also be seen from the structure of pure gauge fluctuations $D \mapsto U D U^{*}$ with $U=u J u J^{-1}$ and $u \in \mathcal{U}(\mathcal{A})$. Indeed, in the absence of the first order condition we find that

$$
U D U^{*}=u\left[D, u^{*}\right]+\hat{u}\left[D, \hat{u}^{*}\right]+\hat{u}\left[u\left[D, u^{*}\right], \hat{u}^{*}\right] .
$$

In the above prescription this corresponds to taking as a universal one-form $A=u \delta\left(u^{*}\right)$.

On a fluctuated Dirac operator $D^{\prime}$ such gauge transformation act in a similar way as $D^{\prime} \mapsto U D^{\prime} U^{*}$. By construction, it is implemented by the gauge transformation

$$
A \mapsto u A u^{*}+u \delta\left(u^{*}\right)
$$

in the universal differential calculus. In particular, this implies that

$$
A_{(1)} \mapsto u A_{(1)} u^{*}+u\left[D, u^{*}\right]
$$

so the first-order inner fluctuations transform as usual. For the term $A_{(2)}$ we compute that a gauge transformation acts as

$$
A_{(2)} \mapsto J u J^{-1} A_{(2)} J u^{*} J^{-1}+J u J^{-1}\left[u\left[D, u^{*}\right], J u^{*} J^{-1}\right]
$$

where the $A_{(2)}$ on the right-hand-side is expressed using the gauge transformed $A_{(1)}$. This non-linear gauge transformation for $A_{(2)}$ confirms our interpretation of $A_{(2)}$ as the nonlinear contribution to the inner fluctuations.

It turns out [13] that inner fluctuations come from the action on operators in Hilbert space of a semi-group $\operatorname{Pert}(\mathcal{A})$ of inner perturbations which only depends on the involutive algebra $\mathcal{A}$ and extends the unitary group of $\mathcal{A}$. More precisely, the semi-group $\operatorname{Pert}(\mathcal{A})$ consists of normalized self-adjoint elements in $\mathcal{A} \otimes \mathcal{A}^{\mathrm{op}}$ :

$$
\operatorname{Pert}(\mathcal{A}):=\left\{\sum_{j} a_{j} \otimes b_{j}^{\mathrm{op}} \in \mathcal{A} \otimes \mathcal{A}^{\mathrm{op}}: \sum_{j} a_{j} b_{j}=1, \quad \sum_{j} a_{j} \otimes b_{j}^{\mathrm{op}}=\sum_{j} b_{j}^{*} \otimes a_{j}^{* \mathrm{op}}\right\}
$$


with $\mathcal{A}^{\mathrm{op}}$ the involutive algebra $\mathcal{A}$ but with the opposite product $(a b)^{\mathrm{op}}=b^{\mathrm{op}} a^{\mathrm{op}}$. The semi-group product is inherited from the multiplication in the algebra $\mathcal{A} \otimes \mathcal{A}^{\mathrm{op}}$, that is:

$$
\left(\sum_{i} a_{i} \otimes b_{i}^{\mathrm{op}}\right)\left(\sum_{j} a_{j}^{\prime} \otimes\left(b_{j}^{\prime}\right)^{\mathrm{op}}\right)=\sum_{i, j} a_{i} a_{j}^{\prime} \otimes\left(b_{j}^{\prime} b_{i}\right)^{\mathrm{op}},
$$

which indeed respects the above normalization and self-adjointness condition. Note that the unitary group of $\mathcal{A}$ is mapped to $\operatorname{Pert}(\mathcal{A})$ by sending a unitary $u$ to $u \otimes u^{* \mathrm{op}}$.

Given a spectral triple $(\mathcal{A}, \mathcal{H}, D)$ an inner fluctuation of $D$ by an element $\sum_{j} a_{j} \otimes b_{j}^{\text {op }}$ in $\operatorname{Pert}(\mathcal{A})$ is now simply given by

$$
D \mapsto \sum_{j} a_{j} D b_{j}
$$

This covers both cases of ordinary spectral triples and real spectral triples (i.e. those which are equipped with the operator $J$ ). In the latter case one simply uses the natural homomorphism of semi-groups $\mu: \operatorname{Pert}(\mathcal{A}) \rightarrow \operatorname{Pert}(\mathcal{A} \otimes \hat{\mathcal{A}})$ given by $\mu(A)=A \otimes \hat{A}$. Explicitly, this implies for real spectral triples the following transformation rule:

$$
D \mapsto \sum_{i . j} a_{i} \hat{a}_{j} D b_{i} \hat{b}_{j}
$$

which can indeed be shown [13, Proposition 5] to coincide with the above (2.2).

The structure of a semi-group implies in particular that inner fluctuations of inner fluctuations are still inner fluctuations - a fact which is not at all direct when looking at Equation (2.2) - and that the corresponding algebraic rules are unchanged by passing from ordinary spectral triples to real spectral triples.

\section{Classification of finite geometries without first order condition}

Some time ago the question of classifying finite noncommutative spaces was carried out in [9]. The main restriction came from requiring that spinors which belong to the product of the continuous four dimensional space, times the finite space must be such that the conjugate spinor is not an independent field, in order to avoid doubling the fermions. This could only be achieved when the spinors satisfy both the Majorana and Weyl conditions, which implies that the $K O$-dimension of the finite space be $6(\bmod 8)$. Consistency with the zeroth order condition

$$
\left[a, b^{\circ}\right]=0, \quad b^{\circ}=J b^{*} J^{-1}, \forall a, b \in \mathcal{A}
$$

(since $\mathcal{A}$ is an involutive algebra this condition is the same if one replaces $b^{\circ}$ by $\hat{b}=J b J^{-1}$ ) restricts the center of the complexified algebra to be $Z\left(\mathcal{A}_{\mathbb{C}}\right)=\mathbb{C} \oplus \mathbb{C}$. The dimension of the Hilbert space is then restricted to be the square of an integer. The algebra is then of the form

$$
M_{k}(\mathbb{C}) \oplus M_{k}(\mathbb{C})
$$


A symplectic symmetry imposed on the first algebra forces $k$ to be even $k=2 a$ and the algebra to be of quaternionic matrices of the form $M_{a}(\mathbb{H})$. The existence of the chirality operator breaks $M_{a}(\mathbb{H})$ and further restricts the integer $a$ to be even, and thus the number of fundamental fermions must be of the form $4 a^{2}$ where $a$ is an even integer. This shows that the first possible realistic case is the finite space with $k=4$ to be based on the algebra

$$
\mathcal{A}=\mathbb{H}_{R} \oplus \mathbb{H}_{L} \oplus M_{4}(\mathbb{C}) .
$$

A further restriction arises from the first order condition requiring the commutation of the commutator $[D, a]$ where $D$ is the Dirac operator and $a \in \mathcal{A}$ with elements $b^{\circ}, b \in \mathcal{A}$,

$$
\left[[D, a], b^{\circ}\right]=0, \quad a, b \in \mathcal{A}, \quad b^{\circ}=J b^{*} J^{-1}
$$

( since $\mathcal{A}$ is an involutive algebra this condition is the same if one replaces $b^{\circ}$ by $\hat{b}=J b J^{-1}$ ) This condition, together with the requirement that the neutrinos must acquire a Majorana mass restricts the above algebra further to the subalgebra

$$
\mathbb{C} \oplus \mathbb{H} \oplus M_{3}(\mathbb{C}) .
$$

The question is whether the first order condition is an essential requirement for noncommutative spaces. There are known examples of noncommutative spaces where the first order condition is not satisfied such as the quantum group $\mathrm{SU}(2)_{q}([16,17])$. As recalled in the previous section, the main novelty of not imposing the first order condition is that the fluctuations of the Dirac operator (gauge and Higgs fields) will not be linear anymore and part of it $A_{(2)}$ will depend quadratically on the fields appearing in $A_{(1)}$. In this work we shall study the resulting noncommutative space without imposing the first order condition on the Dirac operator. Our starting point, however, will be an initial Dirac operator (without fluctuations) satisfying the first order condition relative to the subalgebra (3.2), but inner fluctuations would spoil this property.

The noncommutative geometric setting provided answers to some of the basic questions about the SM, such as the number of fermions in one family, the nature of the gauge symmetries and their fields, the fermionic representations, the Higgs fields as gauge fields along discrete directions, the phenomena of spontaneous symmetry breaking as well many other explanations [10]. In other words, noncommutative geometry successfully gave a geometric setting for the SM. The dynamics of the model was then determined by the spectral action principle which is based on the idea that all the geometric invariants of the space can be found in the spectrum of the Dirac operator of the associated space. Indeed it was shown that the spectral action, which is a function of the Dirac operator, can be computed and gives the action of the SM coupled to gravity valid at some high energy scale. When the couplings appearing in this action are calculated at low energies by running the RG equations one finds excellent agreement with all known results to within few percents.

The first order condition is what restricted a more general gauge symmetry based on the algebra $\mathbb{H}_{R} \oplus \mathbb{H}_{L} \oplus M_{4}(\mathbb{C})$ to the subalgebra $\mathbb{C} \oplus \mathbb{H} \oplus M_{3}(\mathbb{C})$. It is thus essential to understand the physical significance of such a requirement. In what follows we shall examine the more general algebra allowed without the first order condition, and shall show 
that the number of fundamental fermions is still dictated to be 16 . We determine the inner automorphisms of the algebra $\mathcal{A}$ and show that the resulting gauge symmetry is a Pati-Salam type left-right model

$$
\mathrm{SU}(2)_{R} \times \mathrm{SU}(2)_{L} \times \mathrm{SU}(4)
$$

where SU (4) is the color group with the lepton number as the fourth color. In addition we observe that the Higgs fields appearing in $A_{(2)}$ are composite and depend quadratically on those appearing in $A_{(1)}$ provided that the initial Dirac operator (without fluctuations) satisfies the order one condition relative to the subalgebra (3.2). Otherwise, there will be additional fundamental Higgs fields. In particular, the representations of the fundamental Higgs fields when the initial Dirac operator satisfies the order one condition are $\left(2_{R}, 2_{L}, 1\right)$, $\left(2_{R}, 1_{L}, 4\right)$ and $\left(1_{R}, 1_{L}, 1+15\right)$ with respect to $\mathrm{SU}(2)_{R} \times \mathrm{SU}(2)_{L} \times \mathrm{SU}(4)$. When such an order one condition is not satisfied for the initial Dirac operator, the representations of the additional Higgs fields are $\left(3_{R}, 1_{L}, 10\right),\left(1_{R}, 1_{L}, 6\right)$ and $\left(2_{R}, 2_{L}, 1+15\right)$. There are simplifications if the Yukawa coupling of the up quark is equated with that of the neutrino and of the down quark equated with that of the electron. In addition the $1+15$ of SU (4) decouple if we assume that at unification scale there is exact SU (4) symmetry between the quarks and leptons. The resulting model is very similar to the one considered by Marshak and Mohapatra [20].

\section{Summary of tensor notation}

Although it is possible to use matrix notation to deal with the physical model, the fact that the matrix representation (which is a product of matrices) is $384 \times 384$ dimensional making the task daunting and not very transparent, although only involving products of matrices. We find it much more efficient and practical to use a tensorial notation which simplifies greatly the algebraic operations. This also has the added advantage of allowing to check all the steps using computer programs with algebraic manipulations such as Mathematica and Maple.

We will restrict to the case where $Z\left(\mathcal{A}_{\mathbb{C}}\right)=\mathbb{C} \oplus \mathbb{C}$. An element of the Hilbert space $\Psi \in \mathcal{H}$ is represented by

$$
\Psi_{M}=\left(\begin{array}{c}
\psi_{A} \\
\psi_{A^{\prime}}
\end{array}\right), \quad \psi_{A^{\prime}}=\psi_{A}^{c}
$$

where $\psi_{A}^{c}$ is the conjugate spinor to $\psi_{A}$. Thus all primed indices $A^{\prime}$ correspond to the Hilbert space of conjugage spinors. It is acted on by both the left algebra $M_{2}(\mathbb{H})$ and the right algebra $M_{4}(\mathbb{C})$. Therefore the index $A$ can take 16 values and is represented by

$$
A=\alpha I
$$

where the index $\alpha$ is acted on by quaternionic matrices and the index $I$ by $M_{4}(\mathbb{C})$ matrices. Moreover, when grading breaks $M_{2}(\mathbb{H})$ into $\mathbb{H}_{R} \oplus \mathbb{H}_{L}$ the index $\alpha$ is decomposed to $\alpha=\dot{a}, a$ where $\dot{a}=\dot{1}, \dot{2}$ (dotted index) is acted on by the first quaternionic algebra $\mathbb{H}_{R}$ and $a=1,2$ is acted on by the second quaternionic algebra $\mathbb{H}_{L}$. When $M_{4}(\mathbb{C})$ breaks into $\mathbb{C} \oplus M_{3}(\mathbb{C})$ 
(due to symmetry breaking or through the use of the order one condition) the index $I$ is decomposed into $I=1, i$ where the 1 is acted on by the $\mathbb{C}$ and the $i$ by $M_{3}(\mathbb{C})$. Therefore the various components of the spinor $\psi_{A}$ are

$$
\begin{aligned}
\psi_{\alpha I} & =\left(\begin{array}{cccc}
\nu_{R} & u_{i R} & \nu_{L} & u_{i L} \\
e_{R} & d_{i R} & e_{L} & d_{i L}
\end{array}\right) \\
& =\left(\psi_{\dot{a} 1}, \psi_{a i}, \psi_{a 1}, \psi_{a i}\right), \quad a=1,2, \quad a=\dot{1}, \dot{2}, \quad i=1,2,3 .
\end{aligned}
$$

The power of the abstract notation can be seen by noting that the Dirac action takes the very simple form

$$
\Psi_{M}^{*} D_{M}^{N} \Psi_{N}
$$

which could be expanded to give

$$
\psi_{A}^{*} D_{A}^{B} \psi_{B}+\psi_{A^{\prime}}^{*} D_{A^{\prime}}^{B} \psi_{B}+\psi_{A}^{*} D_{A}^{B^{\prime}} \psi_{B^{\prime \prime}}+\psi_{A^{\prime}}^{*} D_{A^{\prime}}^{B^{\prime}} \psi_{B^{\prime}}
$$

The Dirac operator can be written in matrix form

$$
D=\left(\begin{array}{cc}
D_{A}^{B} & D_{A}^{B^{\prime}} \\
D_{A^{\prime}}^{B} & D_{A^{\prime}}^{B^{\prime}}
\end{array}\right)
$$

where

$$
\begin{aligned}
& A=\alpha I, \quad \alpha=1, \cdots, 4, \quad I=1, \cdots, 4 \\
& A^{\prime}=\alpha^{\prime} I^{\prime}, \quad \alpha^{\prime}=1^{\prime}, \cdots, 4^{\prime}, \quad I=1^{\prime}, \cdots, 4^{\prime}
\end{aligned}
$$

Thus $D_{A}^{B}=D_{\alpha I}^{\beta J}$. Elements of the algebra

$$
\mathcal{A}=M_{4}(\mathbb{C}) \oplus M_{4}(\mathbb{C})
$$

are represented by

$$
a=\left(\begin{array}{cc}
X_{\alpha}^{\beta} \delta_{I}^{J} & 0 \\
0 & \delta_{\alpha^{\prime}}^{\beta^{\prime}} Y_{I^{\prime}}^{J^{\prime}}
\end{array}\right)
$$

where the first block is the tensor product of elements of $M_{4}(\mathbb{C}) \otimes 1_{4}$ and the second blcok is the tensor product of elements of $1_{4} \otimes M_{4}(\mathbb{C})$. The reality operator $J$ is anti-linear and interchange the first and second blocks and satsify $J^{2}=1$. It is represented by

$$
J=\left(\begin{array}{cc}
0 & \delta_{\alpha}^{\beta^{\prime}} \delta_{I}^{J^{\prime}} \\
\delta_{\alpha^{\prime}}^{\beta} \delta_{I^{\prime}}^{J} & 0
\end{array}\right) \times \text { complex conjugation }
$$

In this form

$$
a^{o}=J a^{*} J^{-1}=\left(\begin{array}{cc}
\delta_{\alpha}^{\beta} Y_{I}^{t J} & 0 \\
0 & X_{\alpha^{\prime}}^{t \beta^{\prime}} \delta_{I^{\prime}}^{J^{\prime}}
\end{array}\right)
$$

where the superscript $t$ denotes the transpose matrix. This clearly satisfies the commutation relation

$$
\left[a, b^{o}\right]=0
$$


Writing

$$
b=\left(\begin{array}{cc}
Z_{\alpha}^{\beta} \delta_{I}^{J} & 0 \\
0 & \delta_{\alpha^{\prime}}^{\beta^{\prime}} W_{I^{\prime}}^{J^{\prime}}
\end{array}\right)
$$

then

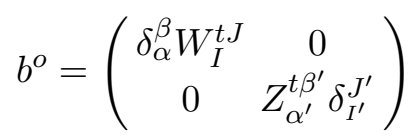

and so $\left[[D, a], b^{o}\right]$ is equal to

$$
\left(\begin{array}{cc}
{\left[[D, X], W^{t}\right]_{A}^{B}} & \left((D Y-X D) Z^{t}-W^{t}(D Y-X D)\right)_{A}^{B^{\prime}} \\
\left((D X-Y D) W^{t}-Z^{t}(D X-Y D)\right)_{A^{\prime}}^{B} & {\left[[D, Y], Z^{t}\right]_{A^{\prime}}^{B^{\prime}}}
\end{array}\right)
$$

The order one condition is

$$
\left[[D, a], b^{o}\right]=0
$$

which admits a solution with non-zero mixing between primed and unprimed indices such as

$$
D_{\alpha I}^{\beta^{\prime} K^{\prime}}=\delta_{\alpha}^{1} \delta_{1^{\prime}}^{\beta^{\prime}} \delta_{I}^{1} \delta_{1^{\prime}}^{K^{\prime}} k^{* \nu_{R}}
$$

only when $a, b$ are restricted to the subalgebra $\mathbb{C} \oplus \mathbb{H} \oplus M_{3}(\mathbb{C}) \subset \mathcal{A}$. Here the $k^{* \nu_{R}}$ are matrices in generation space which will be assumed to be $3 \times 3$. We also note that the property that $D J=J D$ implies that

$$
D_{A^{\prime}}^{B^{\prime}}=\bar{D}_{A}^{B}
$$

We further impose the condition of symplectic isometry on the first $M_{4}(\mathbb{C})$

$$
\left(\sigma_{2} \otimes 1\right)(\bar{a})\left(\sigma_{2} \otimes 1\right)=a, \quad a \in M_{4}(\mathbb{C})
$$

which reduces $M_{4}(\mathbb{C})$ to $M_{2}(\mathbb{H})$. From the property of commutation of the grading operator $G_{\alpha}^{\beta}$ with $M_{2}(\mathbb{H})$

$$
[G, X]=0
$$

where $G_{\alpha}^{\beta}=\left(\begin{array}{cc}1_{2} & 0 \\ 0 & -1_{2}\end{array}\right)$, reduces the algebra $M_{2}(\mathbb{H})$ to $\mathbb{H}_{R} \oplus \mathbb{H}_{L}$. Thus we now have

$$
X_{\alpha}^{\beta}=\left(\begin{array}{cc}
X_{\dot{a}}^{\dot{b}} & 0 \\
0 & X_{a}^{b}
\end{array}\right), \quad X_{a}^{b}=\left(\begin{array}{cc}
X_{1}^{1} & X_{1}^{2} \\
-X_{1}^{2} & X_{1}^{1}
\end{array}\right) \in \mathbb{H}_{L}
$$

and similarly for $X_{\dot{a}}^{\dot{b}} \in \mathbb{H}_{R}$. In matrix form the operator $D_{F}$ has the sub-matrices [10]

$$
\begin{aligned}
D_{\alpha 1}{ }^{\beta 1} & =\left(\begin{array}{cc}
0 & D_{a 1}^{\dot{b} 1} \\
D_{\dot{a} 1}^{b 1} & 0
\end{array}\right), & D_{a 1}^{\dot{b} 1}=\left(D_{\dot{a} 1}^{b 1}\right)^{*} \equiv D_{a(l)}^{\dot{b}} \\
D_{\alpha i}{ }^{\beta j} & =\left(\begin{array}{cc}
0 & D_{a(q)}^{\dot{b}} \delta_{i}^{j} \\
D_{\dot{a}(q)}^{b} \delta_{i}^{j} & 0
\end{array}\right), & D_{\dot{a}(q)}^{b}=\left(D_{a(q)}^{\dot{b}}\right)^{*}
\end{aligned}
$$


where

$$
D_{a 1}^{\dot{b} 1}=D_{a(l)}^{\dot{b}}=\left(\begin{array}{cc}
k^{* \nu} & 0 \\
0 & k^{* e}
\end{array}\right), \quad a=1,2, \quad \dot{b}=\dot{1}, \dot{2}
$$

and

$$
D_{a(q)}^{\dot{b}}=\left(\begin{array}{cc}
k^{* u} & 0 \\
0 & k^{* d}
\end{array}\right) .
$$

The Yukawa couplings $k^{\nu}, k^{e}, k^{u}, k^{d}$ are $3 \times 3$ matrices in generation space. Notice that this structure gives Dirac masses to all the fermions, but Majorana masses only for the right-handed neutrinos. This was shown in [9] to be the unique possibility consistent with the first order condition on the subalgebra (3.2). We can summarize all the information about the finite space Dirac operator without fluctuations, in the tensorial equation

$$
\begin{aligned}
&\left(D_{F}\right)_{\alpha I}^{\beta J}=\left(\delta_{\alpha}^{1} \delta_{\dot{1}}^{\beta} k^{* \nu}+\delta_{\alpha}^{1} \delta_{1}^{\beta} k^{\nu}+\delta_{\alpha}^{2} \delta_{\dot{2}}^{\beta} k^{* e}+\delta_{\alpha}^{2} \delta_{2}^{\beta} k^{e}\right) \delta_{I}^{1} \delta_{1}^{J} \\
&+\left(\delta_{\alpha}^{1} \delta_{\dot{1}}^{\beta} k^{* u}+\delta_{\alpha}^{1} \delta_{1}^{\beta} k^{u}+\delta_{\alpha}^{2} \delta_{\dot{2}}^{\beta} k^{* d}+\delta_{\alpha}^{2} \delta_{2}^{\beta} k^{d}\right) \delta_{I}^{i} \delta_{j}^{J} \delta_{i}^{j} \\
&\left(D_{F}\right)_{\alpha I}^{\beta^{\prime} K^{\prime}=} \delta_{\alpha}^{1} \delta_{i^{\prime}}^{\beta^{\prime}} \delta_{I}^{1} \delta_{1^{\prime}}^{K^{\prime}} k^{* \nu_{R}}
\end{aligned}
$$

where $k^{\nu_{R}}$ are Yukawa couplings for the right-handed neutrinos. One can also consider the special case of lepton and quark unification by equating

$$
k^{\nu}=k^{u}, \quad k^{e}=k^{d}
$$

where we expect some simplifications.

\section{Dirac operator and inner fluctuations on $\mathbb{H}_{R} \oplus \mathbb{H}_{L} \oplus M_{4}(\mathbb{C})$}

Recall that if one considers inner fluctuations of the Dirac operator one finds that the gauge transformation takes the form

$$
D_{A} \rightarrow U D_{A} U^{*}, \quad U=u J u J^{-1}, \quad u \in \mathcal{U}(\mathcal{A})
$$

which implies that

$$
A \rightarrow u A u^{*}+u \delta\left(u^{*}\right)
$$

This in turn gives

$$
\begin{aligned}
& A_{(1)} \rightarrow u A_{(1)} u^{*}+u\left[D, u^{*}\right] \\
& A_{(2)} \rightarrow J u J^{-1} A_{(2)} J u^{*} J^{-1}+J u J^{-1}\left[u\left[D, u^{*}\right], J u^{*} J^{-1}\right]
\end{aligned}
$$

where the $A_{(2)}$ in the right hand side is computed using the gauge transformed $A_{(1)}$. Thus $A_{(1)}$ is a one-form and behaves like the usual gauge transformations. On the other hand $A_{(2)}$ transforms non-linearly and includes terms with quadratic dependence on the gauge transformations.

We now proceed to compute the Dirac operator on the product space $M \times F$. The initial operator is given by

$$
D=\gamma^{\mu} D_{\mu} \otimes 1+\gamma_{5} D_{F}
$$


where $\gamma^{\mu} D_{\mu}=\gamma^{\mu}\left(\partial_{\mu}+\frac{1}{4} \omega_{\mu}{ }^{a b} \gamma_{a b}\right)$ is the Dirac operator on the four dimensional spin manifold. Then the Dirac operator including inner fluctuations is given by

$$
\begin{aligned}
D_{A} & =D+A_{(1)}+J A_{(1)} J^{-1}+A_{(2)} \\
A_{(1)} & =\sum a[D, b] \\
A_{(2)} & =\sum a\left[J A_{(1)} J^{-1}, b\right] .
\end{aligned}
$$

The computation is very involved thus for clarity we shall collect all the details in the appendix and only quote the results in what follows. The different components of the operator $D_{A}$ are then given by

$$
\begin{aligned}
& \left(D_{A}\right)_{\dot{a} I}^{\dot{b} J}=\gamma^{\mu}\left(D_{\mu} \delta_{\dot{a}}^{\dot{b}} \delta_{I}^{J}-\frac{i}{2} g_{R} W_{\mu R}^{\alpha}\left(\sigma^{\alpha}\right)_{\dot{a}}^{\dot{b}} \delta_{I}^{J}-\delta_{\dot{a}}^{\dot{b}}\left(\frac{i}{2} g V_{\mu}^{m}\left(\lambda^{m}\right)_{I}^{J}+\frac{i}{2} g V_{\mu} \delta_{I}^{J}\right)\right) \\
& \left(D_{A}\right)_{a I}^{b J}=\gamma^{\mu}\left(D_{\mu} \delta_{a}^{b} \delta_{I}^{J}-\frac{i}{2} g_{L} W_{\mu L}^{\alpha}\left(\sigma^{\alpha}\right)_{a}^{b} \delta_{I}^{J}-\delta_{a}^{b}\left(\frac{i}{2} g V_{\mu}^{m}\left(\lambda^{m}\right)_{I}^{J}+\frac{i}{2} g V_{\mu} \delta_{I}^{J}\right)\right)
\end{aligned}
$$

where the fifteen $4 \times 4$ matrices $\left(\lambda^{m}\right)_{I}^{J}$ are traceless and generate the group $\mathrm{SU}(4)$ and $W_{\mu R}^{\alpha}, W_{\mu L}^{\alpha}, V_{\mu}^{m}$ are the gauge fields of $\mathrm{SU}(2)_{R}, \mathrm{SU}(2)_{L}$, and $\mathrm{SU}(4)$. The requirement that $A$ is unimodular implies that

$$
\operatorname{Tr}(A)=0
$$

which gives the condition

$$
V_{\mu}=0
$$

In addition we have

$$
\begin{aligned}
\left(D_{A}\right)_{\dot{a} I}^{b J} & =\gamma_{5}\left(\left(k^{\nu} \phi_{\dot{a}}^{b}+k^{e} \widetilde{\phi}_{\dot{a}}^{b}\right) \Sigma_{I}^{J}+\left(k^{u} \phi_{\dot{a}}^{b}+k^{d} \widetilde{\phi}_{\dot{a}}^{b}\right)\left(\delta_{I}^{J}-\Sigma_{I}^{J}\right)\right) \equiv \gamma_{5} \Sigma_{\dot{a} I}^{b J} \\
\left(D_{A}\right)_{\dot{a} I}^{\dot{b}^{\prime} J^{\prime}} & =\gamma_{5} k^{* \nu_{R}} \Delta_{\dot{a} J} \Delta_{\dot{b} I} \equiv \gamma_{5} H_{\dot{a} I \dot{b} J}
\end{aligned}
$$

where the Higgs field $\phi_{\dot{a}}^{b}$ is in the $\left(2_{R}, \overline{2}_{L}, 1\right)$ of the product gauge group $\mathrm{SU}(2)_{R} \times \mathrm{SU}(2)_{L} \times$ $\mathrm{SU}(4)$, and $\Delta_{\dot{a} J}$ is in the $\left(2_{R}, 1_{L}, 4\right)$ representation while $\Sigma_{I}^{J}$ is in the $\left(1_{R}, 1_{L}, 1+15\right)$ representation. The field $\widetilde{\phi}_{\dot{a}}^{b}$ is not an independent field and is given by

$$
\widetilde{\phi}_{\dot{a}}^{b}=\sigma_{2} \bar{\phi}_{\dot{a}}^{b} \sigma_{2}
$$

Note that the field $\Sigma_{I}^{J}$ decouples (and set to $\delta_{I}^{1} \delta_{1}^{J}$ ) in the special case when there is lepton and quark unification of the couplings

$$
k^{\nu}=k^{u}, \quad k^{e}=k^{d} .
$$

In case when the initial Dirac operator satisfies the order one condition for the subalgebra (3.2), then the $A_{(2)}$ part of the connection becomes a composite Higgs field where the Higgs field $\Sigma_{\dot{a} I}^{b J}$ is formed out of the products of the fields $\phi_{\dot{a}}^{b}$ and $\Sigma_{I}^{J}$ while the Higgs field $H_{\dot{a} I \dot{b} J}$ is made from the product of $\Delta_{\dot{a} J} \Delta_{\dot{b} I}$. For generic initial Dirac operators, the 
field $\left(A_{(2)}\right)_{\dot{a} I}^{b J}$ becomes independent. The fields $\Sigma_{\dot{a} I}^{b J}$ and $H_{\dot{a} I \dot{b} J}$ will then not be defined through equation 5.1 and will be in the $\left(2_{R}, 2_{L}, 1+15\right)$ and $\left(3_{R}, 1_{L}, 10\right)+\left(1_{R}, 1_{L}, 6\right)$ representations of $\mathrm{SU}(2)_{R} \times \mathrm{SU}(2)_{L} \times \mathrm{SU}(4)$. In addition, for generic Dirac operator one also generates the fundamental field $\left(1,2_{L}, 4\right)$. The fact that inner automorphisms form a semigroup implies that the cases where the Higgs fields contained in the connections $A_{(2)}$ are either independent fields or depend quadratically on the fundamental Higgs fields are disconnected. The interesting question that needs to be addressed is whether the structure of the connection is preserved at the quantum level. This investigation must be performed in such a way as to take into account the noncommutative structure of the space. At any rate, we have here a clear advantage over grand unified theories which suffers of having arbitrary and complicated Higgs representations. In the noncommutative geometric setting, this problem is now solved by having minimal representations of the Higgs fields. Remarkably, we note that a very close model to the one deduced here is the one considered by Marshak and Mohapatra where the $U(1)$ of the left-right model is identified with the $B-L$ symmetry. They proposed the same Higgs fields that would result starting with a generic initial Dirac operator not satisfying the first order condition. Although the broken generators of the SU (4) gauge fields can mediate lepto-quark interactions leading to proton decay, it was shown that in all such types of models with partial unification, the proton is stable. In addition this type of model arises in the first phase of breaking of $S O(10)$ to $\mathrm{SU}(2)_{R} \times \mathrm{SU}(2)_{L} \times \mathrm{SU}(4)$ and these have been extensively studied [1]. The recent work in [18] considers noncommutative grand unification based on the $k=8$ algebra $M_{4}(\mathbb{H}) \oplus M_{8}(\mathbb{C})$ keeping the first order condition.

\section{The spectral action for the $\mathrm{SU}(2)_{R} \times \mathrm{SU}(2)_{L} \times \mathrm{SU}(4)$ model}

Having determined the Dirac operator acting on the Hilbert space of spinors in terms of the gauge fields of SU $(2)_{R} \times \mathrm{SU}(2)_{L} \times \mathrm{SU}(4)$ and Higgs fields, some of which are fundamental while others are composite, the next step is to study the dynamics of these fields as governed by the spectral action principle. The geometric invariants of the noncommutative space are encoded in the spectrum of the Dirac operator $D_{A}$. The bosonic action is given by

$$
\text { Trace }\left(f\left(D_{A} / \Lambda\right)\right)
$$

where $\Lambda$ is some cutoff scale and the function $f$ is restricted to be even and positive. Using heat kernel methods the trace can be expressed in terms of Seeley-de Witt coefficients $a_{n}$ :

$$
\text { Trace } f\left(D_{A} / \Lambda\right)=\sum_{n=0}^{\infty} F_{4-n} \Lambda^{4-n} a_{n}
$$

where the function $F$ is defined by $F(u)=f(v)$ where $u=v^{2}$, thus $F\left(D^{2}\right)=f(D)$. We define

$$
f_{k}=f(v) v^{k-1} d v, \quad k>0
$$


then

$$
\begin{aligned}
F_{4} & =\int_{0}^{\infty} F(u) u d u=2 \int_{0}^{\infty} f(v) v^{3} d v=2 f_{4} \\
F_{2} & =\int_{0}^{\infty} F(u) d u=2 \int_{0}^{\infty} f(v) v d v=2 f_{2} \\
F_{0} & =F(0)=f(0)=f_{0} \\
F_{-2 n} & =(-1)^{n} F^{(n)}(0)=\left[(-1)^{n}\left(\frac{1}{2 v} \frac{d}{d v}\right)^{n} f\right](0) \quad n \geq 1 .
\end{aligned}
$$

Using the same notation and formulas as in reference [10], the first Seeley-de Witt coefficient is

$$
\begin{aligned}
a_{0} & =\frac{1}{16 \pi^{2}} \int d^{4} x \sqrt{g} \operatorname{Tr}(1) \\
& =\frac{1}{16 \pi^{2}}(4)(32)(3) \int d^{4} x \sqrt{g} \\
& =\frac{24}{\pi^{2}} \int d^{4} x \sqrt{g}
\end{aligned}
$$

where the numerical factors come, respectively, from the traces on the Clifford algebra, the dimensions of the Hilbert space and number of generations. The second coefficient is

$$
a_{2}=\frac{1}{16 \pi^{2}} \int d^{4} x \sqrt{g} \operatorname{Tr}\left(E+\frac{1}{6} R\right)
$$

where $E$ is a $384 \times 384$ matrix over Hilbert space of three generations of spinors, whose components are derived and listed in the appendix. Taking the various traces we get

$$
\begin{aligned}
a_{2} & =\frac{1}{16 \pi^{2}} \int d^{4} x \sqrt{g}\left(\left(R(-96+64)-8\left(H_{\dot{a} I \dot{c} K} H^{\dot{c} K \dot{a} I}+2 \Sigma_{\dot{a} I}^{c K} \Sigma_{c K}^{\dot{a} I}\right)\right)\right. \\
& =-\frac{2}{\pi^{2}} \int d^{4} x \sqrt{g}\left(R+\frac{1}{4}\left(H_{\dot{a} I \dot{c} K} H^{\dot{c} K \dot{a} I}+2 \Sigma_{\dot{a} I}^{c K} \Sigma_{c K}^{\dot{a} I}\right)\right) .
\end{aligned}
$$

It should be understood in the above formula and in what follows, that whenever the matrices $k^{\nu}, k^{u}, k^{e}, k^{d}$ and $k^{\nu_{R}}$ appear in an action, one must take the trace over generation space. When the initial Dirac operator without fluctuations is taken to satisfy the order one condition, the fields $H_{\dot{a} I \dot{c} K}$ and $\Sigma_{\dot{a} I}^{c K}$ will become dependent on the fundamental Higgs fields. In this case, the mass terms can be expressed in terms of the fundamental Higgs field to give

$$
H_{\dot{a} I \dot{c} K} H^{\dot{c} K \dot{a} I}=\left|k^{\nu_{R}}\right|^{2}\left(\Delta_{\dot{a} K} \bar{\Delta}^{\dot{a} K}\right)^{2}
$$

and

$$
\begin{aligned}
2 \Sigma_{\dot{a} I}^{c K} \Sigma_{c K}^{\dot{a} I}=2( & \left.\left(\left(k^{\nu}-k^{u}\right) \phi_{\dot{a}}^{c}+\left(k^{e}-k^{d}\right) \widetilde{\phi}_{\dot{a}}^{c}\right) \Sigma_{I}^{K}+\left(k^{u} \phi_{\dot{a}}^{c}+k^{d} \widetilde{\phi}_{\dot{a}}^{c}\right) \delta_{I}^{K}\right) \\
& \left(\left(\left(k^{* \nu}-k^{* u}\right) \phi_{c}^{\dot{a}}+\left(k^{* e}-k^{* d}\right) \widetilde{\phi}_{c}^{\dot{a}}\right) \Sigma_{K}^{I}+\left(k^{* u} \phi_{c}^{\dot{a}}+k^{* d} \widetilde{\phi}_{c}^{\dot{a}}\right) \delta_{K}^{I}\right) .
\end{aligned}
$$


The next coefficient is

$$
a_{4}=\frac{1}{16 \pi^{2}} \int d^{4} x \sqrt{g} \operatorname{Tr}\left(\frac{1}{360}\left(5 R^{2}-2 R_{\mu \nu}^{2}+2 R_{\mu \nu \rho \sigma}^{2}\right) 1+\frac{1}{2}\left(E^{2}+\frac{1}{3} R E+\frac{1}{6} \Omega_{\mu \nu}^{2}\right)\right)
$$

where $\Omega_{\mu \nu}$ is the $384 \times 384$ curvature matrix of the connection $\omega_{\mu}$. Using the expressions for the matrices $E$ and $\Omega_{\mu \nu}$ derived in the appendix, and taking the traces, we get

$$
\begin{aligned}
a_{4}=\frac{1}{2 \pi^{2}} \int & d^{4} x \sqrt{g}\left[-\frac{3}{5} C_{\mu \nu \rho \sigma}^{2}+\frac{11}{30} R^{*} R^{*}+g_{L}^{2}\left(W_{\mu \nu L}^{\alpha}\right)^{2}+g_{R}^{2}\left(W_{\mu \nu R}^{\alpha}\right)^{2}+g^{2}\left(V_{\mu \nu}^{m}\right)^{2}\right. \\
& +\nabla_{\mu} \Sigma_{a I}^{\dot{c} K} \nabla^{\mu} \Sigma_{\dot{c} K}^{a I}+\frac{1}{2} \nabla_{\mu} H_{\dot{a} I \dot{b} J} \nabla^{\mu} H^{\dot{a} I \dot{b} J}+\frac{1}{12} R\left(H_{\dot{a} I \dot{c} K} H^{\dot{c} K \dot{a} I}+2 \Sigma_{\dot{a} I}^{c K} \Sigma_{c K}^{\dot{a} I}\right) \\
& \left.+\frac{1}{2}\left|H_{\dot{a} I \dot{c} K} H^{\dot{c} K \dot{b} J}\right|^{2}+2 H_{\dot{a} I \dot{c} K} \Sigma_{b J}^{\dot{c} K} H^{\dot{a} I \dot{d} L} \sum_{\dot{d} L}^{b J}+\Sigma_{a I}^{\dot{c} K} \sum_{\dot{c} K}^{b J} \Sigma_{b J}^{\dot{d} L} \Sigma_{\dot{d} L}^{a I}\right]
\end{aligned}
$$

where $C_{\mu \nu \rho \sigma}$ is the Weyl tensor. Thus the bosonic spectral action to second order is given by

$$
S=F_{4} \Lambda^{4} a_{0}+F_{2} \Lambda^{2} a_{2}+F_{0} a_{4}+\cdots
$$

which finally gives

$$
\begin{aligned}
S_{\mathrm{b}}= & \frac{24}{\pi^{2}} F_{4} \Lambda^{4} \int d^{4} x \sqrt{g} \\
& -\frac{2}{\pi^{2}} F_{2} \Lambda^{2} \int d^{4} x \sqrt{g}\left(R+\frac{1}{4}\left(H_{\dot{a} I \dot{c} K} H^{\dot{c} K \dot{a} I}+2 \Sigma_{\dot{a} I}^{c K} \Sigma_{c K}^{\dot{a} I}\right)\right) \\
& +\frac{1}{2 \pi^{2}} F_{0} \int d^{4} x \sqrt{g}\left[\frac{1}{30}\left(-18 C_{\mu \nu \rho \sigma}^{2}+11 R^{*} R^{*}\right)+g_{L}^{2}\left(W_{\mu \nu L}^{\alpha}\right)^{2}+g_{R}^{2}\left(W_{\mu \nu R}^{\alpha}\right)^{2}+g^{2}\left(V_{\mu \nu}^{m}\right)^{2}\right. \\
& +\nabla_{\mu} \Sigma_{a I}^{\dot{c} K} \nabla^{\mu} \Sigma_{\dot{c} K}^{a I}+\frac{1}{2} \nabla_{\mu} H_{\dot{a} I \dot{b} J} \nabla^{\mu} H^{\dot{a} I \dot{b} J}+\frac{1}{12} R\left(H_{\dot{a} I \dot{c} K} H^{\dot{c} K \dot{a} I}+2 \Sigma_{\dot{a} I}^{c K} \Sigma_{c K}^{\dot{a} I}\right) \\
& \left.+\frac{1}{2}\left|H_{\dot{a} I \dot{c} K K} H^{\dot{c} K \dot{b} J}\right|^{2}+2 H_{\dot{a} I \dot{c} K K} \Sigma_{b J}^{\dot{c} K} H^{\dot{a} I \dot{d} L} \sum_{\dot{d} L}^{b J}+\Sigma_{a I}^{\dot{c} K} \sum_{\dot{c} K}^{b J} \Sigma_{b J}^{\dot{d} L} \Sigma_{\dot{d} L}^{a I}\right] .
\end{aligned}
$$

The physical content of this action is a cosmological constant term, the Einstein Hilbert term $R$, a Weyl tensor square term $C_{\mu \nu \rho \sigma}^{2}$, kinetic terms for the $\mathrm{SU}(2)_{R} \times \mathrm{SU}(2)_{L} \times \mathrm{SU}(4)$ gauge fields, kinetic terms for the composite Higgs fields $H_{\dot{a} I \dot{b} J}$ and $\Sigma_{b J}^{\dot{c} K}$ as well as mass terms and quartic terms for the Higgs fields. This is a grand unified Pati-Salam type model with a completely fixed Higgs structure which we expect to spontaneously break at very high energies to the $U(1) \times \mathrm{SU}(2) \times \mathrm{SU}(3)$ symmetry of the SM. We also notice that this action gives the gauge coupling unification

$$
g_{R}=g_{L}=g .
$$

A test of this model is to check whether this relation when run using RG equations would give values consistent with the values of the gauge couplings for electromagnetic, weak and strong interactions at the scale of the $Z$-boson mass. Having determined the full Dirac operators, including fluctuations, we can write all the fermionic interactions including the 
ones with the gauge vectors and Higgs scalars. It is given by

$$
\begin{aligned}
\int d^{4} x & \sqrt{g}\left\{\psi_{\dot{a} I}^{*} \gamma^{\mu}\left(D_{\mu} \delta_{\dot{a}}^{\dot{b}} \delta_{I}^{J}-\frac{i}{2} g_{R} W_{\mu R}^{\alpha}\left(\sigma^{\alpha}\right)_{\dot{a}}^{\dot{b}} \delta_{I}^{J}-\delta_{\dot{a}}^{\dot{b}}\left(\frac{i}{2} g V_{\mu}^{m}\left(\lambda^{m}\right)_{I}^{J}+\frac{i}{2} g V_{\mu} \delta_{I}^{J}\right)\right) \psi_{\dot{b} J}\right. \\
& +\psi_{a I}^{*} \gamma^{\mu}\left(D_{\mu} \delta_{a}^{b} \delta_{I}^{J}-\frac{i}{2} g_{L} W_{\mu L}^{\alpha}\left(\sigma^{\alpha}\right)_{a}^{b} \delta_{I}^{J}-\delta_{a}^{b}\left(\frac{i}{2} g V_{\mu}^{m}\left(\lambda^{m}\right)_{I}^{J}+\frac{i}{2} g V_{\mu} \delta_{I}^{J}\right)\right) \psi_{b J} \\
& \left.+\psi_{\dot{a} I}^{*} \gamma_{5} \Sigma_{\dot{a} I}^{b J} \psi_{b J}+\psi_{a I}^{*} \gamma_{5} \Sigma_{a I}^{\dot{b} J} \psi_{\dot{b} J}+C \psi_{\dot{a} I} \gamma_{5} H^{\dot{a} I \dot{b} J} \psi_{\dot{b} J}+\text { h.c. }\right\}
\end{aligned}
$$

\section{Truncation to the standard model}

It is easy to see that this model truncates to the Standard Model. The Higgs field $\phi_{\dot{a}}^{b}$ $=\left(2_{R}, 2_{L}, 1\right)$ must be truncated to the Higgs doublet $H$ by writing

$$
\phi_{\dot{a}}^{b}=\delta_{\dot{a}}^{\dot{1}} \epsilon^{b c} H_{c} .
$$

The other Higgs field $\Delta_{\dot{a} I}=\left(2_{R}, 1,4\right)$ is truncated to a real singlet scalar field

$$
\Delta_{\dot{a} I}=\delta_{\dot{a}}^{1} \delta_{I}^{1} \sqrt{\sigma}
$$

These then imply the relations

$$
\begin{aligned}
\Sigma_{\dot{a} I}^{b J} & =\left(\delta_{\dot{a}}^{\dot{1}} k^{\nu} \epsilon^{b c} H_{c}+\delta_{\dot{a}}^{\dot{2}} \bar{H}^{b} k^{e}\right) \delta_{I}^{1} \delta_{1}^{J}+\left(\delta_{\dot{a}}^{\dot{1}} k^{u} \epsilon^{b c} H_{c}+\delta_{\dot{a}}^{\dot{2}} k^{d} \bar{H}^{b}\right) \delta_{I}^{i} \delta_{j}^{J} \delta_{i}^{j} \\
H_{\dot{a} I \dot{b} J} & =\delta_{\dot{a}}^{\dot{1}} \delta_{\dot{b}}^{\dot{1}} k^{\nu_{R}} \delta_{I}^{1} \delta_{1}^{J} \sigma \\
g_{R} W_{\mu R}^{3} & =g_{1} B_{\mu}, \quad W_{\mu R}^{ \pm}=0 \\
\sqrt{\frac{3}{2}} g V_{\mu}^{15} & =-g_{1} B_{\mu} \quad\left(V_{\mu}\right)_{1}^{i}=0
\end{aligned}
$$

where $V_{\mu}^{15}$ is the $\mathrm{SU}(4)$ gauge field corresponding to the generator

$$
\lambda^{15}=\frac{1}{\sqrt{6}} \operatorname{diag}(3,-1,-1,-1)
$$

which could be identified with the $B-L$ generator. In particular the components $\left(D_{A}\right)_{i 1}^{11}$ and $\left(D_{A}\right)_{\dot{2} 1}^{\dot{21}}$ of the Dirac operator simplify to

$$
\begin{aligned}
\left(D_{A}\right)_{\dot{11}}^{\mathrm{i} 1} & =\gamma^{\mu}\left(D_{\mu}-\frac{i}{2} g_{R} W_{\mu R}^{\alpha}\left(\sigma^{\alpha}\right)_{\dot{1}}^{\mathrm{i}}-\left(\frac{i}{2} g V_{\mu}^{m}\left(\lambda^{m}\right)_{1}^{1}\right)\right) \\
& =\gamma^{\mu}\left(D_{\mu}-\frac{i}{2} g_{R} W_{\mu R}^{3}-\left(\frac{i}{2} g V_{\mu}^{15} \sqrt{\frac{3}{2}}\right)\right) \\
& =\gamma^{\mu} D_{\mu} \\
\left(D_{A}\right)_{\dot{2} 1}^{21} & =\gamma^{\mu}\left(D_{\mu}-\frac{i}{2} g_{R} W_{\mu R}^{\alpha}\left(\sigma^{\alpha}\right)_{\dot{2}}^{\dot{2}}-\left(\frac{i}{2} g V_{\mu}^{m}\left(\lambda^{m}\right)_{1}^{1}\right)\right) \\
& =\gamma^{\mu}\left(D_{\mu}+\frac{i}{2} g_{R} W_{\mu R}^{3}-\left(\frac{i}{2} g V_{\mu}^{15} \sqrt{\frac{3}{2}}\right)\right) \\
& =\gamma^{\mu}\left(D_{\mu}+i g_{1} B_{\mu}\right)
\end{aligned}
$$


which are identified with the Dirac operators acting on the right-handed neutrino and right-handed electron. Similar substitutions give the action of the Dirac operators on the remaining fermions and give the expected results. We now compute the various terms in the spectral action. First for the mass terms we have

$$
\begin{aligned}
\frac{1}{4} H_{\dot{a} I \dot{b} J} H^{\dot{b J a} I} & =\frac{1}{4}\left(\delta_{\dot{a}}^{1} \delta_{\dot{b}}^{1} k^{\nu_{R}} \delta_{I}^{1} \delta_{1}^{J} \sigma\right)\left(\delta_{1}^{\dot{a}} \delta_{1}^{\dot{b}} \delta_{1}^{I} \delta_{1}^{J} k^{* \nu_{R}} \sigma\right) \\
& =\frac{1}{4} \operatorname{tr}\left|k^{\nu_{R}}\right|^{2} \sigma^{2}=\frac{1}{4} c \sigma^{2} \\
\frac{1}{2} \Sigma_{\dot{a} I}^{c K} \Sigma_{c K}^{\dot{a} I} & =\frac{1}{2}\left|\left(\delta_{\dot{a}}^{\dot{1}} k^{\nu} \epsilon^{b c} H_{c}+\delta_{\dot{a}}^{2} \bar{H}^{b} k^{e}\right) \delta_{I}^{1} \delta_{1}^{J}+\left(\delta_{\dot{a}}^{\dot{1}} k^{u} \epsilon^{b c} H_{c}+\delta_{\dot{a}}^{\dot{2}} k^{d} \bar{H}^{b}\right) \delta_{I}^{i} \delta_{j}^{J} \delta_{i}^{j}\right|^{2} \\
& =\frac{1}{2} a \bar{H} H
\end{aligned}
$$

where

$$
\begin{aligned}
& a=\operatorname{tr}\left(k^{* \nu} k^{\nu}+k^{* e} k^{e}+3\left(k^{* u} k^{u}+k^{* d} k^{d}\right)\right) \\
& c=\operatorname{tr}\left(k^{* \nu_{R}} k^{\nu_{R}}\right)
\end{aligned}
$$

Next for the $a_{4}$ term, starting with the gauge kinetic energies we have

$$
g_{L}^{2}\left(W_{\mu \nu L}^{\alpha}\right)^{2}+g_{R}^{2}\left(W_{\mu \nu R}^{\alpha}\right)^{2}+g^{2}\left(V_{\mu \nu}^{m}\right)^{2} \rightarrow g_{L}^{2}\left(W_{\mu \nu L}^{\alpha}\right)^{2}+\frac{5}{3} g_{1}^{2} B_{\mu \nu}^{2}+g_{3}^{2}\left(V_{\mu \nu}^{m}\right)^{2}
$$

where $m=1, \cdots, 8$ for $V_{\mu \nu}^{m}$ restricted to the $\mathrm{SU}(3)$ gauge group. Next for the Higgs kinetic and quartic terms we have

$$
\begin{aligned}
\nabla_{\mu} \Sigma_{a I}^{\dot{c} K} \nabla^{\mu} \Sigma_{\dot{c} K}^{a I} & \rightarrow a \nabla_{\mu} \bar{H} \nabla^{\mu} H \\
\frac{1}{2} \nabla_{\mu} H_{\dot{a} I \dot{b} J} \nabla^{\mu} H^{\dot{a} I \dot{b} J} & \rightarrow \frac{1}{2} c \partial_{\mu} \sigma \partial^{\mu} \sigma \\
\frac{1}{12} R\left(H_{\dot{a} I \dot{c} K} H^{\dot{c} K \dot{a} I}+2 \Sigma_{\dot{a} I}^{c K} \Sigma_{c K}^{\dot{a} I}\right) & \rightarrow \frac{1}{12} R\left(2 a \bar{H} H+c \sigma^{2}\right) \\
\frac{1}{2}\left|H_{\dot{a} I \dot{c} K} H^{\dot{c} K \dot{b} J}\right|^{2} & \rightarrow \frac{1}{2} d \sigma^{4} \\
2 H_{\dot{a} I \dot{c} K} \Sigma_{b J}^{\dot{c} K} H^{\dot{a} I \dot{d} L} \sum_{\dot{d} L}^{b J} & \rightarrow 2 e \bar{H} H \sigma^{2} \\
\Sigma_{a I}^{\dot{c} K} \sum_{\dot{c} K}^{b J} \Sigma_{b J}^{\dot{d} L} \Sigma_{\dot{d} L}^{a I} & \rightarrow b(\bar{H} H) .{ }^{2}
\end{aligned}
$$

Collecting all terms we end up with the bosonic action for the Standard Model:

$$
\begin{aligned}
S_{\mathrm{b}}= & \frac{24}{\pi^{2}} F_{4} \Lambda^{4} \int d^{4} x \sqrt{g} \\
& -\frac{2}{\pi^{2}} F_{2} \Lambda^{2} \int d^{4} x \sqrt{g}\left(R+\frac{1}{2} a \bar{H} H+\frac{1}{4} c \sigma^{2}\right) \\
& +\frac{1}{2 \pi^{2}} F_{0} \int d^{4} x \sqrt{g}\left[\frac{1}{30}\left(-18 C_{\mu \nu \rho \sigma}^{2}+11 R^{*} R^{*}\right)+\frac{5}{3} g_{1}^{2} B_{\mu \nu}^{2}+g_{2}^{2}\left(W_{\mu \nu}^{\alpha}\right)^{2}+g_{3}^{2}\left(V_{\mu \nu}^{m}\right)^{2}\right. \\
& \left.\quad+\frac{1}{6} a R \bar{H} H+b(\bar{H} H)^{2}+a\left|\nabla_{\mu} H_{a}\right|^{2}+2 e \bar{H} H \sigma^{2}+\frac{1}{2} d \sigma^{4}+\frac{1}{12} c R \sigma^{2}+\frac{1}{2} c\left(\partial_{\mu} \sigma\right)^{2}\right]
\end{aligned}
$$


where

$$
\begin{aligned}
& b=\operatorname{tr}\left(\left(k^{* \nu} k^{\nu}\right)^{2}+\left(k^{* e} k^{e}\right)^{2}+3\left(\left(k^{* u} k^{u}\right)^{2}+\left(k^{* d} k^{d}\right)^{2}\right)\right) \\
& d=\operatorname{tr}\left(\left(k^{* \nu_{R}} k^{\nu_{R}}\right)^{2}\right) \\
& e=\operatorname{tr}\left(k^{* \nu} k^{\nu} k^{* \nu_{R}} k^{\nu_{R}}\right) .
\end{aligned}
$$

This action completely agrees with the results in reference [10].

\section{The potential and symmetry breaking}

We now study the resulting potential and try to investigate the possible minima:

$$
\begin{aligned}
V & =\frac{F_{0}}{2 \pi^{2}}\left(\frac{1}{2}\left|H_{\dot{a} I \dot{c} K} H^{\dot{c} K \dot{b} J}\right|^{2}+2 H_{\dot{a} I \dot{c} K} \sum_{b J}^{\dot{c} K} H^{\dot{a} I \dot{d} L} \sum_{\dot{d} L}^{b J}+\Sigma_{a I}^{\dot{c} K} \sum_{\dot{c} K}^{b J} \sum_{b J}^{\dot{d} L} \sum_{\dot{d} L}^{a I}\right) \\
& -\frac{F_{2}}{2 \pi^{2}}\left(H_{\dot{a} I \dot{c} K} H^{\dot{c} K \dot{a} I}+2 \Sigma_{\dot{a} I}^{c K} \Sigma_{c K}^{\dot{a} I}\right) .
\end{aligned}
$$

However, the Higgs field here are not fundamental and we have to express the potential in terms of the fundamental Higgs fields $\phi_{\dot{a}}^{c}, \Delta_{\dot{a} K}$ and $\Sigma_{K}^{I}$. Expanding the composite Higgs fields in terms of the fundamental ones, we have for the quartic terms

$$
\begin{aligned}
\frac{1}{2}\left|H_{\dot{a} I \dot{c} K} H^{\dot{c} K \dot{b} J}\right|^{2}= & \frac{1}{2}\left|k^{\nu_{R}}\right|^{4}\left(\Delta_{\dot{a} K} \bar{\Delta}^{\dot{a} L} \Delta_{\dot{b} L} \bar{\Delta}^{\dot{b} K}\right)^{2} \\
\Sigma_{a I}^{\dot{c} K} \Sigma_{\dot{c} K}^{b J} \Sigma_{b J}^{\dot{d} L} \Sigma_{\dot{d} L}^{a I}= & \left.\left(\left(k^{* \nu}-k^{* u}\right) \phi_{a}^{\dot{c}}+\left(k^{* e}-k^{* d}\right) \widetilde{\phi}_{a}^{\dot{c}}\right) \Sigma_{I}^{K}+\left(k^{* u} \phi_{a}^{\dot{c}}+k^{* d} \widetilde{\phi}_{a}^{\dot{c}}\right) \delta_{I}^{K}\right) \\
& \left(\left(\left(k^{\nu}-k^{u}\right) \phi_{\dot{c}}^{b}+\left(k^{e}-k^{d}\right) \widetilde{\phi}_{\dot{c}}^{b}\right) \Sigma_{K}^{J}+\left(k^{u} \phi_{\dot{c}}^{b}+k^{d} \widetilde{\phi}_{\dot{c}}^{b}\right) \delta_{K}^{J}\right) \\
& \left(\left(\left(k^{* \nu}-k^{* u}\right) \phi_{b}^{\dot{d}}+\left(k^{* e}-k^{* d}\right) \widetilde{\phi}_{b}^{\dot{d}}\right) \Sigma_{J}^{L}+\left(k^{* u} \phi_{b}^{\dot{d}}+k^{* d} \widetilde{\phi}_{b}^{d}\right) \delta_{J}^{L}\right) \\
& \left(\left(\left(k^{\nu}-k^{u}\right) \phi_{\dot{d}}^{a}+\left(k^{e}-k^{d}\right) \widetilde{\phi}_{\dot{d}}^{a}\right) \Sigma_{L}^{I}+\left(k^{u} \phi_{\dot{d}}^{a}+k^{d} \widetilde{\phi}_{\dot{d}}^{a}\right) \delta_{L}^{I}\right) \\
2 H_{\dot{a} I \dot{c} K} \Sigma_{b J}^{\dot{c} K} H^{\dot{a} I \dot{d} L} \Sigma_{\dot{d} L}^{b J}=2 \mid & \left|k^{\nu_{R}}\right|^{2}\left(\Delta_{\dot{a} K} \bar{\Delta}^{\dot{a} L} \Delta_{\dot{c} I} \bar{\Delta}^{\dot{d} I}\right) \\
& \left(\left(\left(k^{* \nu}-k^{* u}\right) \phi_{b}^{\dot{c}}+\left(k^{* e}-k^{* d}\right) \widetilde{\phi}_{b}^{\dot{c}}\right) \Sigma_{J}^{K}+\left(k^{* u} \dot{\phi}_{b}^{\dot{c}}+k^{* d} \widetilde{\phi}_{b}^{\dot{c}}\right) \delta_{J}^{K}\right) \\
& \left(\left(\left(k^{\nu}-k^{u}\right) \phi_{\dot{d}}^{b}+\left(k^{e}-k^{d}\right) \widetilde{\phi}_{\dot{d}}^{b}\right) \Sigma_{L}^{J}+\left(k^{u} \phi_{\dot{d}}^{b}+k^{d} \widetilde{\phi}_{\dot{d}}^{b}\right) \delta_{L}^{J}\right) .
\end{aligned}
$$

Next we have the mass terms

$$
H_{\dot{a} I \dot{c} K} H^{\dot{c} K \dot{a} I}=\left|k^{\nu_{R}}\right|^{2}\left(\Delta_{\dot{a} K} \bar{\Delta}^{\dot{a} K}\right)^{2}
$$

and

$$
\begin{aligned}
2 \Sigma_{\dot{a} I}^{c K} \Sigma_{c K}^{\dot{a} I}=2 & \left(\left(\left(k^{\nu}-k^{u}\right) \phi_{\dot{a}}^{c}+\left(k^{e}-k^{d}\right) \widetilde{\phi}_{\dot{a}}^{c}\right) \Sigma_{I}^{K}+\left(k^{u} \phi_{\dot{a}}^{c}+k^{d} \widetilde{\phi}_{\dot{a}}^{c}\right) \delta_{I}^{K}\right) \\
& \left(\left(\left(k^{* \nu}-k^{* u}\right) \phi_{c}^{\dot{a}}+\left(k^{* e}-k^{* d}\right) \widetilde{\phi}_{c}^{\dot{a}}\right) \Sigma_{K}^{I}+\left(k^{* u} \phi_{c}^{\dot{a}}+k^{* d} \widetilde{\phi}_{c}^{\dot{a}}\right) \delta_{K}^{I}\right) .
\end{aligned}
$$



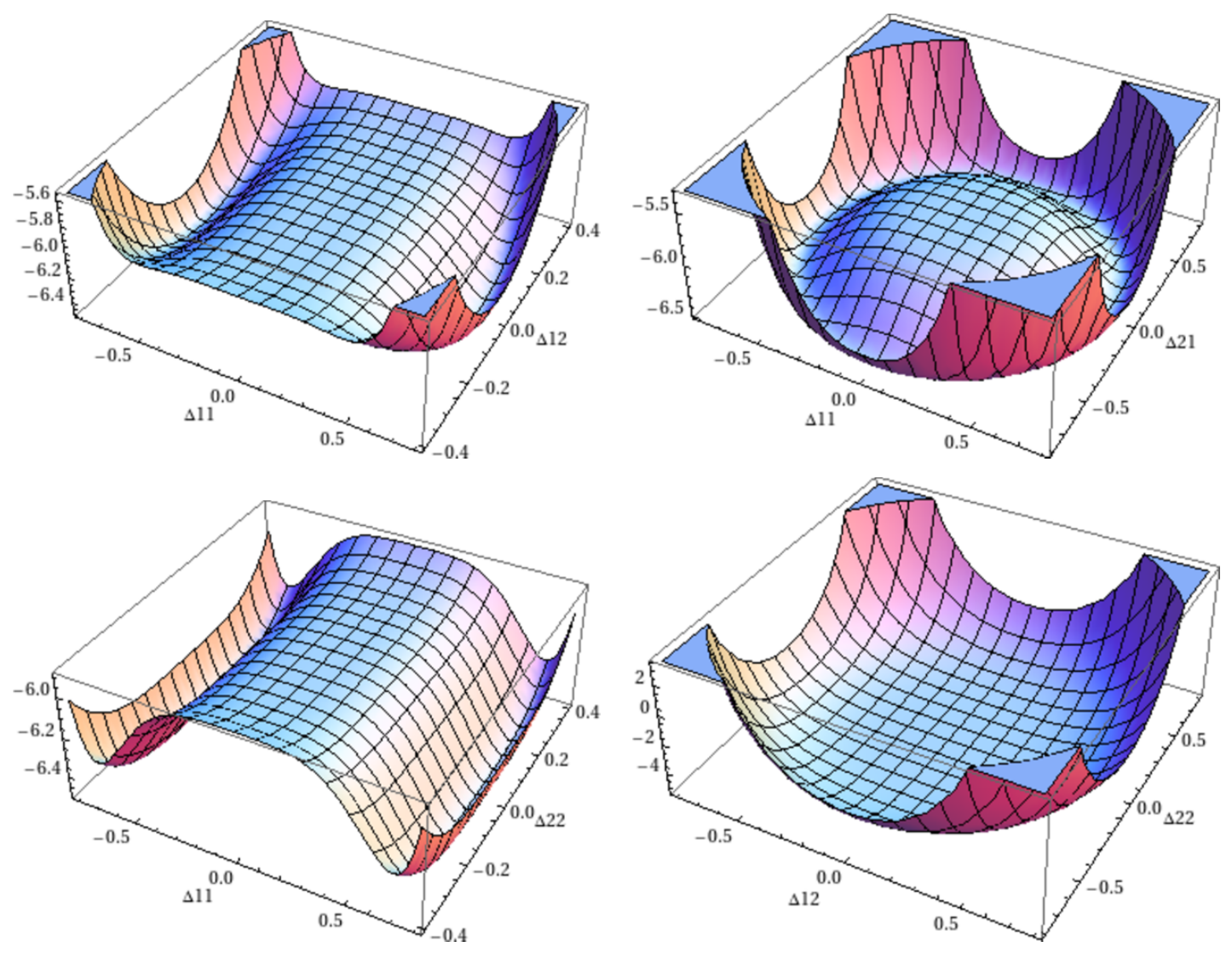

Figure 1. The scalar potential in some of the $\Delta_{\dot{a} I}$-directions, with all other fields at their SM-vevs as in equation (8.1). We have put $k^{\nu}=k^{e}=1$ and $k^{\nu_{R}}=k^{u}=k^{d}=2$. With these choices, the Standard Model vacuum corresponds to $\Delta_{i 1}=\frac{1}{\sqrt{2}}, \Sigma_{1}^{1}=2, \phi_{i}^{1}=\frac{1}{2}$ and all other fields are zero. At this point the Hessian in the $\Delta$-directions is nonnegative.

The potential must be analyzed to determine all the possible minima that breaks the symmetry $\mathrm{SU}(2)_{R} \times \mathrm{SU}(2)_{L} \times \mathrm{SU}(4)$. In this respect it is useful to determine whether the symmetries of this model break correctly at high energies to the Standard Model.

Needless to say that it is difficult to determine all allowed vacua of this potential, especially since there is dependence of order eight on the fields. It is possible, however, to expand this potential around the vacuum that we started with which breaks the gauge symmetry directly from $\mathrm{SU}(2)_{R} \times \mathrm{SU}(2)_{L} \times \mathrm{SU}(4)$ to $U(1)_{\mathrm{em}} \times \mathrm{SU}(3)_{\mathrm{c}}$. Explicitly, this vacuum is given by

$$
\left\langle\phi_{\dot{a}}^{b}\right\rangle=v \delta_{\dot{a}}^{\dot{1}} \delta_{1}^{b} \quad\left\langle\Sigma_{J}^{I}\right\rangle=u \delta_{1}^{I} \delta_{J}^{1} \quad\left\langle\Delta_{\dot{a} J}\right\rangle=w \delta_{\dot{a}}^{\dot{1}} \delta_{J}^{1}
$$

We have included several plots of the scalar potential in the $\Delta_{\dot{a} J}$-directions in figure 1 . A computation of the Hessian in the $\Delta$-directions shows that the SM-vev is indeed a local minimum.

The first order condition now arises as a vacuum solution of the spectral action as follows. We let the $\Delta$-fields take their vev according to the scalar potential, i.e. $\Delta_{a J}=$ $w \delta_{\dot{a}}^{\dot{1}} \delta_{J}^{1}$. Since $\Delta_{\dot{a} J}$ is in the $\left(2_{R}, 1_{L}, 4\right)$ representation of $\mathrm{SU}(2)_{R} \times \mathrm{SU}(2)_{L} \times \mathrm{SU}(4)$, this 

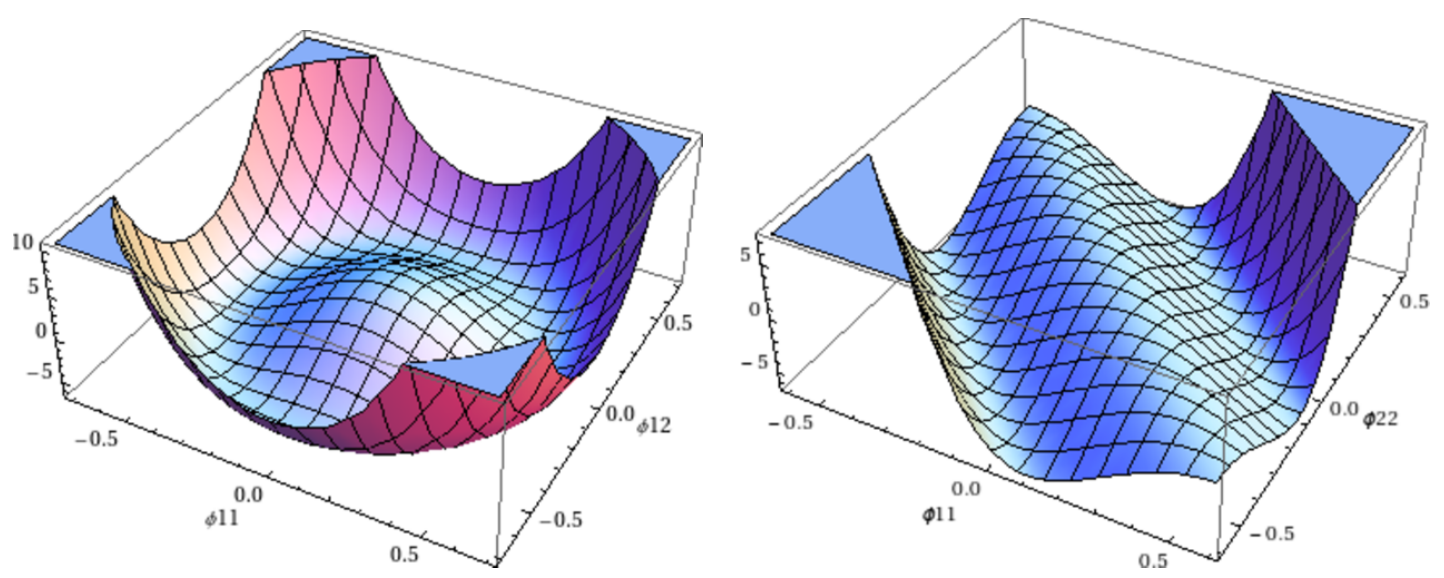

Figure 2. The scalar potential in the $\phi_{\dot{a}}^{b}$-directions, after the $\Sigma$ and $\Delta$-fields have acquired their SM-vevs as in Equation (8.1). Again, we have put $k^{\nu}=k^{e}=1$ and $k^{\nu_{R}}=k^{u}=k^{d}=2$.

vacuum solution is only invariant under the subgroup

$$
\left\{\left(\left(\begin{array}{cc}
\lambda & 0 \\
0 & \lambda
\end{array}\right), u_{L}, \lambda \oplus \lambda^{-1 / 3} u\right): \lambda \in \mathrm{U}(1), u_{L} \in \mathrm{SU}(2), u \in \mathrm{SU}(3)\right\} \subset \mathrm{SU}(2)_{R} \times \mathrm{SU}(2)_{L} \times \mathrm{SU}(4)
$$

This is the spontaneous symmetry breaking to $\mathrm{U}(1) \times \mathrm{SU}(2)_{L} \times \mathrm{SU}(3)_{\mathrm{c}}$, thus selecting the subalgebra (3.2). Note that unimodularity on $\mathcal{U}(\mathcal{A})$ naturally induces unimodularity of the spectral Standard Model, hence it generates the correct hypercharges for the fermions.

After the $\Delta$ and $\Sigma$-fields have acquired their vevs, there is a remaining scalar potential for the $\phi$-fields, which is depicted in figure 2. As with the Standard Model Higgs sector, the selection of a minimum further breaks the symmetry from $\mathrm{U}(1) \times \mathrm{SU}(2)_{L} \times \mathrm{SU}(3)_{c}$ to $\mathrm{U}(1)_{\mathrm{em}} \times \mathrm{SU}(3)_{\mathrm{c}}$. The plot on the right in figure 2 suggests that, instead of the SM-vacuum, the vevs of the $\phi$-fields can also be taken of the form

$$
\left\langle\phi_{\dot{a}}^{b}\right\rangle=v \delta_{\dot{a}}^{\dot{1}} \delta_{1}^{b}+v^{\prime} \delta_{\dot{a}}^{2} \delta_{2}^{b}
$$

Let us see which of the gauge fields acquire non-zero mass after spontaneous symmetry breaking, by expanding around the Standard Model vacuum

$$
\begin{gathered}
\phi_{\dot{a}}^{b}=v \delta_{\dot{a}}^{i} \delta_{1}^{b}+H_{\dot{a}}^{b} \\
\Sigma_{J}^{I}=u \delta_{1}^{I} \delta_{J}^{1}+M_{I}^{J} \\
\Delta_{\dot{a} J}=w \delta_{\dot{a}}^{\dot{1}} \delta_{J}^{1}+N_{\dot{a} J}
\end{gathered}
$$

and keep only terms of up to order 4 . First we look at the kinetic term

$$
\begin{aligned}
\nabla_{\mu} H_{\dot{a} I \dot{b} J} & =\partial_{\mu} H_{\dot{a} I \dot{b} J}-\frac{i}{2} g_{R} W_{\mu R}^{\alpha}\left(\sigma^{\alpha}\right)_{\dot{a}}^{\dot{c}} H_{\dot{c} I \dot{b} J}-\frac{i}{2} g_{R} W_{\mu R}^{\alpha}\left(\sigma^{\alpha}\right)_{\dot{b}}^{\dot{c}} H_{\dot{a} I \dot{c} J} \\
& -\frac{i}{2} g V_{\mu}^{m}\left(\lambda^{m}\right)_{I}^{K} H_{\dot{a} K \dot{b} J}-\frac{i}{2} g V_{\mu}^{m}\left(\lambda^{m}\right)_{J}^{K} H_{\dot{a} I \dot{b} K} \cdot
\end{aligned}
$$

To lowest orders we have

$$
\begin{aligned}
H_{\dot{a} I \dot{b} J} & =\left(k^{* \nu_{R}}\right)^{2}\left(w \delta_{\dot{a}}^{\dot{1}} \delta_{J}^{1}+N_{\dot{a} J}\right)\left(w \delta_{\dot{b}}^{1} \delta_{I}^{1}+N_{\dot{b} I}\right) \\
& =\left(k^{* \nu_{R}}\right)^{2}\left(w^{2} \delta_{\dot{a}}^{\dot{1}} \delta_{J}^{1} \delta_{\dot{b}}^{\dot{1}} \delta_{I}^{1}+w \delta_{\dot{a}}^{1} \delta_{J}^{1} N_{\dot{b} I}+w \delta_{\dot{b}}^{1} \delta_{I}^{1} N_{\dot{a} J}+N_{\dot{a} J} N_{\dot{b} I}\right)
\end{aligned}
$$


and so

$$
\begin{aligned}
& \nabla_{\mu} H_{\dot{a} I \dot{b} J}=\left(k^{* \nu_{R}}\right)^{2} w\left(\delta_{\dot{a}}^{\dot{1}} \delta_{J}^{1} \partial_{\mu} N_{\dot{b} I}+\delta_{\dot{b}}^{\dot{1}} \delta_{I}^{1} \partial_{\mu} N_{\dot{a} J}-\frac{i}{2} g_{R} W_{\mu R}^{\alpha}\left(\sigma^{\alpha}\right)_{\dot{a}}^{\dot{1}} w \delta_{J}^{1} \delta_{\dot{b}}^{\dot{1}} \delta_{I}^{1}\right. \\
& \left.-\frac{i}{2} g_{R} W_{\mu R}^{\alpha}\left(\sigma^{\alpha}\right)_{\dot{b}}^{\dot{1}} w \delta_{\dot{a}}^{\dot{1}} \delta_{J}^{1} \delta_{I}^{1}-\frac{i}{2} g V_{\mu}^{m}\left(\lambda^{m}\right)_{I}^{1} w \delta_{\dot{a}}^{\dot{1}} \delta_{J}^{1} \delta_{\dot{b}}^{\mathrm{i}}-\frac{i}{2} g V_{\mu}^{m}\left(\lambda^{m}\right)_{J}^{1} w \delta_{\dot{a}}^{1} \delta_{\dot{b}}^{1} \delta_{I}^{1}\right) \\
& =\left(k^{* \nu_{R}}\right)^{2} w\left(2 \delta_{\dot{a}}^{1} \delta_{J}^{1} \delta_{\dot{b}}^{1} \delta_{I}^{1} \partial_{\mu} N_{\mathrm{i} 1}+\delta_{\dot{a}}^{\dot{1}} \delta_{J}^{1} \delta_{\dot{b}}^{2} \delta_{I}^{1} \partial_{\mu} N_{\dot{2} 1}+\delta_{\dot{a}}^{\dot{1}} \delta_{J}^{1} \delta_{I}^{i} \delta_{\dot{b}}^{1} \partial_{\mu} N_{\dot{1} i}\right. \\
& +\delta_{\dot{a}}^{1} \delta_{J}^{1} \delta_{I}^{i} \delta_{\dot{b}}^{2} \partial_{\mu} N_{\dot{2} i}+\delta_{\dot{b}}^{1} \delta_{I}^{1} \delta_{\dot{a}}^{\dot{1}} \delta_{J}^{j} \partial_{\mu} N_{\dot{1} j}+\delta_{\dot{b}}^{\dot{1}} \delta_{I}^{1} \delta_{\dot{a}}^{2} \delta_{J}^{1} \partial_{\mu} N_{\dot{2} 1}+\delta_{\dot{b}}^{\dot{1}} \delta_{I}^{1} \delta_{\dot{a}}^{\dot{2}} \delta_{J}^{j} \partial_{\mu} N_{\dot{2} j} \\
& -i g_{R} W_{\mu R}^{3} w \delta_{\dot{a}}^{\dot{1}} \delta_{J}^{1} \delta_{\dot{b}}^{\dot{1}} \delta_{I}^{1}-\frac{i}{2} g_{R} W_{\mu R}^{-} w \delta_{\dot{a}}^{\dot{2}} \delta_{J}^{1} \delta_{\dot{b}}^{\dot{1}} \delta_{I}^{1}-\frac{i}{2} g_{R} W_{\mu R}^{-} \delta_{\dot{b}}^{2} w \delta_{\dot{a}}^{\dot{1}} \delta_{J}^{1} \delta_{I}^{1} \\
& -i\left(g_{R} W_{\mu R}^{3}+g \sqrt{\frac{3}{2}} V_{\mu}^{15}\right) \delta_{\dot{a}}^{i} \delta_{J}^{1} \delta_{\dot{b}}^{i} \delta_{I}^{1}-\frac{i}{2} g V_{\mu}^{m}\left(\lambda^{m}\right)_{i}^{1} w \delta_{\dot{a}}^{\dot{1}} \delta_{J}^{1} \delta_{\dot{b}}^{\dot{1}} \delta_{I}^{i}
\end{aligned}
$$

from which it is clear that if we write

$$
\begin{aligned}
g_{R} W_{\mu R}^{3} & =g_{1} B_{\mu}+g_{1}^{\prime} Z_{\mu}^{\prime} \\
g \sqrt{\frac{3}{2}} V_{\mu}^{15} & =-g_{1} B_{\mu}+g_{1}^{\prime} Z_{\mu}^{\prime}
\end{aligned}
$$

then the vector $B_{\mu}$ will not get a mass term while the fields $W_{\mu R}^{ \pm}, Z_{\mu}^{\prime}, V_{\mu}^{m}\left(\lambda^{m}\right)_{i}^{1}$ (these are the fields in the coset of $\left.\frac{\mathrm{SU}(2)_{R} \times \mathrm{SU}(4)}{\mathrm{SU}(3) \times U(1)}\right)$ will all become massive, with mass of order $w^{2}$ as can be seen from the kinetic term

$$
\begin{aligned}
\nabla_{\mu} \Sigma_{\dot{a} I}^{b J}= & \partial_{\mu} \Sigma_{\dot{a} I}^{b J}-\frac{i}{2} g_{R} W_{\mu R}^{\alpha}\left(\sigma^{\alpha}\right)_{\dot{a}}^{\dot{c}} \Sigma_{\dot{c} I}^{b J}+\frac{i}{2} g_{R} W_{\mu R}^{\alpha}\left(\sigma^{\alpha}\right)_{c}^{b} \Sigma_{\dot{a} I}^{c J} \\
& -\frac{i}{2} g V_{\mu}^{m}\left(\lambda^{m}\right)_{I}^{K} \Sigma_{\dot{a} K}^{b J}+\frac{i}{2} g V_{\mu}^{m}\left(\lambda^{m}\right)_{K}^{J} \Sigma_{\dot{a} I}^{b K} .
\end{aligned}
$$

To lowest orders we have

$$
\begin{aligned}
\Sigma_{\dot{a} I}^{b J}= & \left(\left(\left(k^{\nu}-k^{u}\right) \phi_{\dot{a}}^{b}+\left(k^{e}-k^{d}\right) \widetilde{\phi}_{\dot{a}}^{b}\right) \Sigma_{I}^{J}+\left(k^{u} \phi_{\dot{a}}^{b}+k^{d} \widetilde{\phi}_{\dot{a}}^{b}\right) \delta_{I}^{J}\right) \\
= & \left(\left(k^{\nu}-k^{u}\right)\left(v \delta_{\dot{a}}^{\dot{1}} \delta_{1}^{b}+H_{\dot{a}}^{b}\right)+\left(k^{e}-k^{d}\right)\left(v \delta_{\dot{a}}^{2} \delta_{2}^{b}+\widetilde{H}_{\dot{a}}^{b}\right)\right)\left(u \delta_{1}^{J} \delta_{I}^{1}+M_{I}^{J}\right) \\
& +\left(k^{u}\left(v \delta_{\dot{a}}^{\dot{1}} \delta_{1}^{b}+H_{\dot{a}}^{b}\right)+k^{d}\left(v \delta_{\dot{a}}^{2} \delta_{2}^{b}+\widetilde{H}_{\dot{a}}^{b}\right)\right) \delta_{I}^{J} \\
= & v\left(\left(\left(k^{\nu}-k^{u}\right) \delta_{\dot{a}}^{\dot{1}} \delta_{1}^{b}+\left(k^{e}-k^{d}\right) \delta_{\dot{a}}^{2} \delta_{2}^{b}\right) u \delta_{1}^{J} \delta_{I}^{1}+\left(k^{u} \delta_{\dot{a}}^{\dot{1}} \delta_{1}^{b}+k^{d} \delta_{\dot{a}}^{2} \delta_{2}^{b}\right) \delta_{I}^{J}\right) \\
& +\left(\left(k^{\nu}-k^{u}\right) H_{\dot{a}}^{b}+\left(k^{e}-k^{d}\right) \widetilde{H}_{\dot{a}}^{b}\right) u \delta_{1}^{J} \delta_{I}^{1}+\left(k^{u} H_{\dot{a}}^{b}+k^{d} \widetilde{H}_{\dot{a}}^{b}\right) \delta_{I}^{J} \\
& +v\left(\left(k^{\nu}-k^{u}\right) \delta_{\dot{a}}^{1} \delta_{1}^{b}+\left(k^{e}-k^{d}\right) \delta_{\dot{a}}^{2} \delta_{2}^{b}\right) M_{I}^{J} \\
\nabla_{\mu} \Sigma_{\dot{a} I}^{b J}= & \left(\left(k^{\nu}-k^{u}\right) \partial_{\mu} H_{\dot{a}}^{b}+\left(k^{e}-k^{d}\right) \partial_{\mu} \widetilde{H}_{\dot{a}}^{b}\right) u \delta_{1}^{J} \delta_{I}^{1}+\left(k^{u} \partial_{\mu} H_{\dot{a}}^{b}+k^{d} \partial_{\mu} \widetilde{H}_{\dot{a}}^{b}\right) \delta_{I}^{J} \\
& +v\left(\left(k^{\nu}-k^{u}\right) \delta_{\dot{a}}^{\dot{1}} \delta_{1}^{b}+\left(k^{e}-k^{d}\right) \delta_{\dot{a}}^{2} \delta_{2}^{b}\right) \partial_{\mu} M_{I}^{J} \\
& -\frac{i}{2} v g_{R} W_{\mu R}^{\alpha}\left(\sigma^{\alpha}\right)_{\dot{a}}^{\dot{c}}\left(\left(\left(k^{\nu}-k^{u}\right) \delta_{\dot{c}}^{\dot{1}} \delta_{1}^{b}+\left(k^{e}-k^{d}\right) \delta_{\dot{c}}^{2} \delta_{2}^{b}\right) u \delta_{1}^{J} \delta_{I}^{1}\right. \\
& \left.\quad+\left(k^{u} \delta_{\dot{c}}^{\dot{1}} \delta_{1}^{b}+k^{d} \delta_{\dot{c}}^{2} \delta_{2}^{b}\right) \delta_{I}^{J}\right)
\end{aligned}
$$




$$
\begin{gathered}
+\frac{i}{2} v g_{L} W_{\mu L}^{\alpha}\left(\sigma^{\alpha}\right)_{c}^{b}\left(\left(\left(k^{\nu}-k^{u}\right) \delta_{\dot{a}}^{\dot{1}} \delta_{1}^{c}+\left(k^{e}-k^{d}\right) \delta_{\dot{a}}^{2} \delta_{2}^{c}\right) u \delta_{1}^{J} \delta_{I}^{1}\right. \\
\left.+\left(k^{u} \delta_{\dot{a}}^{\dot{1}} \delta_{1}^{c}+k^{d} \delta_{\dot{a}}^{2} \delta_{2}^{c}\right) \delta_{I}^{J}\right) \\
-\frac{i}{2} v g V_{\mu}^{m}\left(\lambda^{m}\right)_{I}^{K}\left(\left(\left(k^{\nu}-k^{u}\right) \delta_{\dot{a}}^{\dot{1}} \delta_{1}^{b}+\left(k^{e}-k^{d}\right) \delta_{\dot{a}}^{\dot{2}} \delta_{2}^{b}\right) u \delta_{1}^{J} \delta_{K}^{1}\right. \\
\left.+\left(k^{u} \delta_{\dot{a}}^{\dot{1}} \delta_{1}^{b}+k^{d} \delta_{\dot{a}}^{2} \delta_{2}^{b}\right) \delta_{K}^{J}\right) \\
+\frac{i}{2} g v V_{\mu}^{m}\left(\lambda^{m}\right)_{K}^{J}\left(\left(\left(k^{\nu}-k^{u}\right) \delta_{\dot{a}}^{\dot{1}} \delta_{1}^{b}+\left(k^{e}-k^{d}\right) \delta_{\dot{a}}^{\dot{2}} \delta_{2}^{b}\right) u \delta_{1}^{K} \delta_{I}^{1}\right. \\
\left.+\left(k^{u} \delta_{\dot{a}}^{\dot{1}} \delta_{1}^{b}+k^{d} \delta_{\dot{a}}^{2} \delta_{2}^{b}\right) \delta_{I}^{K}\right) .
\end{gathered}
$$

For simplicity we will set $u=1$. Isolating the gauge dependent part

$$
\begin{aligned}
& \nabla_{\mu} \Sigma_{\dot{1} 1}^{11} \supset-\frac{i}{2} v\left(g_{R} W_{\mu R}^{3}-g_{L} W_{\mu L}^{3}\right) k^{\nu} \\
& \nabla_{\mu} \Sigma_{\dot{2}}^{21} \supset \frac{i}{2} v\left(g_{R} W_{\mu R}^{3}-g_{L} W_{\mu L}^{3}\right) k^{e} \\
& \nabla_{\mu} \Sigma_{\dot{1} i}^{11} \supset-\frac{i}{2} v g V_{\mu}^{m}\left(\lambda^{m}\right)_{i}^{1}\left(k^{\nu}-k^{u}\right) \\
& \nabla_{\mu} \Sigma_{\dot{1} i}^{1 j} \supset-\frac{i}{2} v\left(g_{R} W_{\mu R}^{3}-g_{L} W_{\mu L}^{3}\right) k^{u} \delta_{i}^{j} \\
& \nabla_{\mu} \Sigma_{\dot{2} i}^{2 j} \supset \frac{i}{2} v\left(g_{R} W_{\mu R}^{3}-g_{L} W_{\mu L}^{3}\right) k^{d} \delta_{i}^{j} \\
& \nabla_{\mu} \Sigma_{\dot{1}}^{21} \supset-\frac{i}{2} v\left(g_{R} W_{\mu R}^{-}-g_{L} W_{\mu L}^{-}\right) k^{\nu} \\
& \nabla_{\mu} \Sigma_{\dot{2} 1}^{11} \supset-\frac{i}{2} v\left(g_{R} W_{\mu R}^{+}-g_{L} W_{\mu L}^{+}\right) k^{e} \\
& \nabla_{\mu} \Sigma_{\dot{2} i}^{11} \supset 0 .
\end{aligned}
$$

Noticing that $g_{R} W_{\mu R}^{3}-g_{L} W_{\mu L}^{3}=\left(g_{1} B_{\mu}-g_{L} W_{\mu L}^{3}\right)+g_{1}^{\prime} Z_{\mu}^{\prime}$ shows that the $Z_{\mu}$ vector gets a mass of order of the weak scale $g v$ while the $W_{\mu R}^{ \pm}$and $Z_{\mu}^{\prime}$ will get a small correction to its mass of order $g w$. Thus we get the correct gauge breaking pattern with the gauge fields $W_{\mu L}$ and $Z$ of the Standard model having masses of the order of the electroweak scale. It is important, however, to see explicitly that the mixing between the $Z$ and $Z^{\prime}$ vectors and $W_{L}^{ \pm}, W_{R}^{ \pm}$are suppressed.

It remains to minimize the potential to determine all possible minima as well as studying the unified model and check whether it allows for unification of coupling constants

$$
g_{R}=g_{L}=g
$$

in addition to determining the top quark mass and Higgs mass. Obviously, this model deserves careful analysis, which will be the subject of future work.

We conclude that the study of noncommutative spaces based on a product of a continuous four dimensional manifold times a finite space of $K O$-dimension 6 , without the first order condition gives rise to almost unique possibility in the form of a Pati-Salam type model. This provides a setting for unification avoiding the desert and which goes beyond 
the SM. In addition one of the vacua of the Higgs fields gives rise at low energies to a Dirac operator satisfying the first order condition. In this way, the first order condition arises as a spontaneously broken phase of higher symmetry and is not imposed from outside.

\section{A Detailed calculations for the practitioner}

For the benefit of the reader, we shall present in this appendix a detailed derivation of the Dirac operator and the spectral action for the noncommutative space on $\mathbb{H}_{R} \oplus \mathbb{H}_{L} \oplus M_{4}(\mathbb{C})$.

For $A_{(1)}$ we have the definition

$$
\left(A_{(1)}\right)_{M}{ }^{N}=\sum a_{M}^{P}[D, b]_{P}^{N}
$$

where

$$
a_{M}^{N}=\left(\begin{array}{cc}
X_{\alpha}^{\prime \beta} \delta_{I}^{J} & 0 \\
0 & \delta_{\alpha^{\prime}}^{\beta^{\prime}} Y_{I^{\prime}}^{\prime J^{\prime}}
\end{array}\right)
$$

which in terms of components give

$$
\begin{aligned}
\left(A_{(1)}\right)_{\alpha I}^{\beta J} & =\sum a_{\alpha I}^{\gamma K}\left(D_{\gamma K}^{\delta L} b_{\delta L}^{\beta J}-b_{\gamma K}^{\delta L} D_{\delta L}^{\beta J}\right) \\
& =\sum X_{\alpha}^{\prime \gamma}\left(D_{\gamma I}^{\delta J} X_{\delta}^{\beta}-X_{\gamma}^{\delta} D_{\delta I}^{\beta J}\right)
\end{aligned}
$$

where we use the notation for $b$ to be the same as that of $a$ without primes (i.e. $X^{\prime} \rightarrow X$, $Y^{\prime} \rightarrow Y$ ). Since $D_{\alpha I}^{\beta J}$ is non vanishing when connecting a dotted index $\dot{a}$ to $a$, (cf. (4.18)) we have the non-vanishing components

$$
\begin{aligned}
\left(A_{(1)}\right)_{\dot{a} I}^{b J}= & \sum X_{\dot{a}}^{\prime \dot{c}}\left(D_{\dot{c} I}^{d J} X_{d}^{b}-X_{\dot{c}}^{\dot{d}} D_{\dot{d} I}^{b J}\right) \\
= & \delta_{I}^{1} \delta_{1}^{J}\left(\sum X_{\dot{a}}^{\prime c}\left(\left(\delta_{\dot{a}}^{\dot{1}} \delta_{1}^{d} k^{\nu}+\delta_{\dot{a}}^{2} \delta_{2}^{d} k^{e}\right) X_{d}^{b}\right)-X_{\dot{c}}^{\dot{d}}\left(\delta_{\dot{d}}^{\dot{1}} \delta_{1}^{b} k^{\nu}+\delta_{\dot{d}}^{\dot{2}} \delta_{2}^{b} k^{e}\right)\right) \\
& \quad+\delta_{I}^{i} \delta_{j}^{J} \delta_{i}^{j}\left(\sum X_{\dot{a}}^{\prime} \dot{c}\left(\left(\delta_{\dot{a}}^{\dot{1}} \delta_{1}^{b} k^{u}+\delta_{\dot{a}}^{\dot{2}} \delta_{2}^{b} k^{d}\right) X_{d}^{b}\right)-X_{\dot{c}}^{\dot{d}}\left(\delta_{\dot{d}}^{\dot{1}} \delta_{1}^{b} k^{u}+\delta_{\dot{d}}^{2} \delta_{2}^{b} k^{d}\right)\right) \\
& \quad \delta_{I}^{1} \delta_{1}^{J}\left(k^{\nu} \phi_{\dot{a}}^{b}+k^{e} \widetilde{\phi}_{\dot{a}}^{b}\right)+\delta_{I}^{i} \delta_{j}^{J} \delta_{i}^{j}\left(k^{u} \phi_{\dot{a}}^{b}+k^{d} \widetilde{\phi}_{\dot{a}}^{b}\right)
\end{aligned}
$$

where

$$
\begin{aligned}
\phi_{\dot{a}}^{b} & =\sum X_{\dot{a}}^{\prime}{ }^{\dot{1}} X_{1}^{b}-X_{\dot{a}}^{\prime c} X_{\dot{c}}^{\dot{1}} \delta_{1}^{b} \\
\widetilde{\phi}_{\dot{a}}^{b} & =\sum X_{\dot{a}}^{\prime \dot{2}} X_{2}^{b}-X_{\dot{a}}^{\prime c} X_{\dot{c}}^{\dot{2}} \delta_{2}^{b}
\end{aligned}
$$

We can check that

$$
\widetilde{\phi}_{\dot{a}}^{b}=\sigma_{2} \bar{\phi}_{\dot{a}}^{b} \sigma_{2}
$$

For example

$$
\begin{aligned}
\widetilde{\phi}_{\dot{1}}^{1} & =\sum X_{\dot{1}}^{\prime \dot{2}} X_{2}^{1} \\
& =\sum \bar{X}_{\dot{2}}^{\prime} \bar{X}_{1}^{2} \\
& =\bar{\phi}_{\dot{2}}^{2}
\end{aligned}
$$


using the quaternionic property of the $X$. Note that $\phi_{\dot{a}}^{b}$ is in the $\left(2_{R}, 2_{L}, 1\right)$ representation of $\mathrm{SU}(2)_{R} \times \mathrm{SU}(2)_{L} \times \mathrm{SU}(4)$.

Similarly we have

$$
\left(A_{(1)}\right)_{a I}^{\dot{b} J}=\left(\left(A_{(1)}\right)_{\dot{b} J}^{a I}\right)^{*}
$$

(In reality one obtains an expression for $\left(A_{(1)}\right)_{a I}^{\dot{b} J}$ in terms of $\phi_{\dot{a}}^{\prime b}$ which is expressed in terms of the $X$, but the hermiticity of the Dirac operator forces the above relation and imposes a constraint on the $X$.)

Next we have using (4.19)

$$
\begin{aligned}
\left(A_{(1)}\right)_{\alpha I}^{\beta^{\prime} J^{\prime}} & =\sum a_{\alpha I}^{\gamma K}\left(D_{\gamma K}^{\delta^{\prime} L^{\prime}} b_{\delta^{\prime} L^{\prime}}^{\beta^{\prime} J^{\prime}}-b_{\gamma K}^{\delta L} D_{\delta L}^{\beta^{\prime} J \prime}\right) \\
& =\sum X_{\alpha}^{\prime \gamma}\left(D_{\gamma I}^{\beta^{\prime} L^{\prime}} Y_{L^{\prime}}^{J^{\prime}}-X_{\gamma}^{\delta} D_{\delta I}^{\beta^{\prime} J \prime}\right) \\
& =k^{* \nu_{R}} \sum X_{\alpha}^{\prime \gamma}\left(\left(\delta_{\gamma}^{i} \delta_{\dot{1}^{\prime}}^{\beta^{\prime}} \delta_{I}^{1} \delta_{1^{\prime}}^{L^{\prime}}\right) Y_{L^{\prime}}^{J^{\prime}}-X_{\gamma}^{\delta}\left(\delta_{\delta}^{i} \delta_{\dot{i}^{\prime}}^{\beta^{\prime}} \delta_{I}^{1} \delta_{1^{\prime}}^{J^{\prime}}\right)\right) \\
& =k^{* \nu_{R}} \delta_{\alpha}^{\dot{a}} \delta_{{ }_{1}^{\prime}}^{\beta^{\prime}} \delta_{I}^{1} \sum\left(X_{\dot{a}}^{\prime i} Y_{1^{\prime}}^{J^{\prime}}-X_{\dot{a}}^{\dot{c}} X_{\dot{c}}^{\dot{1}} \delta_{1^{\prime}}^{J^{\prime}}\right) \\
\left(A_{(1)}\right)_{\dot{a} I}^{\dot{b}^{\prime} J^{\prime}} & =k^{* \nu_{R}} \delta_{i^{b}}^{\dot{b}^{\prime}} \delta_{I}^{1} \Delta_{\dot{a}}^{J^{\prime}}
\end{aligned}
$$

where

$$
\Delta_{\dot{a}}{ }^{J^{\prime}}=\sum\left(X_{\dot{a}}^{\prime \dot{1}} Y_{1^{\prime}}^{J^{\prime}}-X_{\dot{a}}^{\dot{c}} X_{\dot{c}}^{\dot{1}} \delta_{1^{\prime}}^{J^{\prime}}\right) \equiv \Delta_{\dot{a} J}
$$

which is in the $\left(2_{R}, 1_{L}, 4\right)$ representation of $\mathrm{SU}(2)_{R} \times \mathrm{SU}(2)_{L} \times \mathrm{SU}(4)$. Again, we can compute $\left(A_{(1)}\right)_{\alpha^{\prime} I^{\prime}}^{\beta J}$, which gives a similar expression, but using hermiticity we write

$$
\left(A_{(1)}\right)_{\alpha^{\prime} I^{\prime}}^{\beta J}=\left(\left(A_{(1)}\right)_{\beta J}^{\alpha^{\prime} I^{\prime}}\right)^{*}=k^{\nu_{R}} \delta_{\dot{b}}^{\beta} \delta_{\alpha^{\prime}}^{1^{\prime}} \delta_{1}^{J} \Delta^{\dot{b}} I^{\prime}
$$

In the conjugate space we have

$$
\begin{aligned}
\left(A_{(1)}\right)_{\alpha^{\prime} I^{\prime}}^{\beta^{\prime} J^{\prime}} & =\sum a_{\alpha^{\prime} I^{\prime}}^{\gamma^{\prime} K^{\prime}}\left(D_{\gamma^{\prime} K^{\prime}}^{\delta^{\prime} L^{\prime}} b_{\delta^{\prime} L^{\prime}}^{\beta^{\prime} J^{\prime}}-b_{\gamma^{\prime} K^{\prime}}^{\delta^{\prime} L^{\prime}} D_{\delta^{\prime} L^{\prime}}^{\beta^{\prime} J^{\prime}}\right) \\
& =\sum Y_{I^{\prime}}^{\prime K^{\prime}}\left(D_{\alpha^{\prime} K^{\prime}}^{\beta^{\prime} L^{\prime}} Y_{L^{\prime}}^{J^{\prime}}-Y_{K^{\prime}}^{L^{\prime}} D_{\alpha^{\prime} L^{\prime}}^{\beta^{\prime} J^{\prime}}\right)
\end{aligned}
$$

The only non-vanishing expression would involve a $D$ with mixed $a^{\prime}$ and $\dot{b}^{\prime}$

$$
\begin{aligned}
& \left(A_{(1)}\right)_{\dot{a}^{\prime} I^{\prime}}^{b^{\prime} J^{\prime}}=\sum Y_{I^{\prime}}^{\prime K^{\prime}}\left(D_{\dot{a}^{\prime} K^{\prime}}^{b^{\prime} L^{\prime}} Y_{L^{\prime}}^{J^{\prime}}-Y_{K^{\prime}}^{L^{\prime}} D_{\dot{a}^{\prime} L^{\prime}}^{b^{\prime} J^{\prime}}\right) \\
& =\sum Y_{I^{\prime}}^{K^{\prime}}\left(\left(\delta_{K^{\prime}}^{1^{\prime}} \delta_{1^{\prime}}^{L^{\prime}}\left(\delta_{a^{\prime}}^{i^{\prime}} \delta_{1^{\prime}}^{b^{\prime}} \bar{k}^{\nu}+\delta_{\dot{a}^{\prime}}^{2^{\prime}} \delta_{2^{\prime}}^{b^{\prime}} \bar{k}^{e}\right)+\delta_{K^{\prime}}^{k^{\prime}} \delta_{l^{\prime}}^{L^{\prime}} \delta_{k^{\prime}}^{l^{\prime}}\left(\delta_{a^{\prime}}^{i^{\prime}} \delta_{1^{\prime}}^{b^{\prime}} \bar{k}^{u}+\delta_{\dot{a}^{\prime}}^{2^{\prime}} \delta_{2^{\prime}}^{b^{\prime}} \bar{k}^{d}\right)\right) Y_{L^{\prime}}^{J^{\prime}}\right. \\
& \left.-Y_{K^{\prime}}^{L^{\prime}}\left(\left(\delta_{L^{\prime}}^{1^{\prime}} \delta_{1^{\prime}}^{J^{\prime}}\left(\delta_{a^{\prime}}^{1^{\prime}} \delta_{1^{\prime}}^{b^{\prime}} \bar{k}^{\nu}+\delta_{\dot{a}^{\prime}}^{2^{\prime}} \delta_{2^{\prime}}^{b^{\prime}} \bar{k}^{e}\right)+\delta_{L^{\prime}}^{l^{\prime}} \delta_{j^{\prime}}^{J^{\prime}}{\overline{l^{\prime}}}^{j^{\prime}}\left(\delta_{a^{\prime}}^{i^{\prime}} \delta_{1^{\prime}}^{b^{\prime}}, \bar{k}^{u}+\delta_{\dot{a}^{\prime}}^{2^{\prime}} \delta_{2^{\prime}}^{b^{\prime}} \bar{k}^{d}\right)\right)\right)\right) \\
& =\left(\left(\bar{k}^{\nu}-\bar{k}^{u}\right) \delta_{a^{\prime}}^{\dot{1}^{\prime}} \delta_{1^{\prime}}^{b^{\prime}}+\left(\bar{k}^{e}-\bar{k}^{d}\right) \delta_{a^{\prime}}^{2^{\prime}} \delta_{2^{\prime}}^{b^{\prime}}\right) \Sigma_{I^{\prime}}^{J^{\prime}}
\end{aligned}
$$

where

$$
\Sigma_{I^{\prime}}^{1^{\prime}}=-\sum Y_{I^{\prime}}^{\prime k^{\prime}} Y_{k^{\prime}}^{1^{\prime}}, \quad \Sigma_{I^{\prime}}^{j^{\prime}}=Y_{I^{\prime}}^{\prime 1^{\prime}} Y_{1^{\prime}}^{j^{\prime}}
$$


Notice that if $k^{\nu}=k^{u}$ and $k^{e}=k^{d}$ which is consistent with the picture of having the lepton number as the fourth color then $\Sigma_{I}^{J}$ will decouple. Notice that

$$
\left(A_{(1)}\right)_{a^{\prime} I^{\prime}}^{\dot{b}^{\prime} J^{\prime}}=\left(\left(k^{\nu t}-k^{u t}\right) \delta_{a^{\prime}}^{1^{\prime}} \delta_{\dot{1}^{\prime}}^{\dot{b}^{\prime}}+\left(k^{e t}-k^{d t}\right) \delta_{a^{\prime}}^{2^{\prime}} \delta_{\dot{2}^{\prime}}^{\dot{b}^{\prime}}\right) \Sigma_{I^{\prime}}^{J^{\prime}}
$$

which implies by the hermiticity of

$$
A_{\dot{a}^{\prime} I^{\prime}}^{b^{\prime} J^{\prime}}=\left(A_{b^{\prime} I^{j^{\prime}}}^{\dot{a}^{\prime} I^{\prime}}\right)^{*}
$$

that

$$
\Sigma_{I}^{J}=\left(\Sigma_{I}^{J}\right)^{*}
$$

and thus belong to the $1+15$ representation of $\mathrm{SU}(4)$. There is no indication that the singlet which is equal to the trace $\Sigma_{I}^{I}$ should be absent as there is no apparent identity that equates this trace to zero. In this case we can write

$$
\Sigma_{I}^{J}=\widetilde{\Sigma}_{I}^{J}+\frac{1}{4} \delta_{I}^{J} \Sigma, \quad \Sigma=\Sigma_{I}^{I}, \quad \widetilde{\Sigma}_{I}^{I}=0
$$

Thus at first order we have the Higgs fields $\phi_{a}^{\dot{b}}$ and $\Delta_{\dot{a} I}$. In addition if the Yukawa couplings of the leptons are different from the corresponding quarks (and thus requiring the breaking of the lepton number as the fourth color) then an additional Higgs field $\Sigma_{I}^{J}$ is also generated.

Next it is straightforward to evaluate various components of $J A_{(1)} J^{-1}$ which are given by

$$
\begin{aligned}
\left(J A J^{-1}\right)_{A}^{B} & =\bar{A}_{A^{\prime}}^{B^{\prime}} \\
\left(J A J^{-1}\right)_{A}^{B^{\prime}} & =\bar{A}_{A^{\prime}}^{B} \\
\left(J A J^{-1}\right)_{A^{\prime}}^{B^{\prime}} & =\bar{A}_{A}^{B} \\
\left(J A J^{-1}\right)_{A^{\prime}}^{B} & =\bar{A}_{A}^{B^{\prime}}
\end{aligned}
$$

In particular

$$
\begin{aligned}
\left(J A_{(1)} J^{-1}\right)_{\dot{a} I}^{b J} & =\left(\bar{A}_{(1)}\right)_{\dot{a}^{\prime} I^{\prime}}^{b^{\prime} J^{\prime}} \\
& =\left(\left(k^{\nu}-k^{u}\right) \delta_{\dot{a}}^{\dot{1}} \delta_{1}^{b}+\left(k^{e}-k^{d}\right) \delta_{\dot{a}}^{2} \delta_{2}^{b}\right) \Sigma_{I}^{J t} \\
\left(J A_{(1)} J^{-1}\right)_{\dot{a} I}^{\dot{b}^{\prime} J^{\prime}} & =\left(\bar{A}_{(1)}\right)_{\dot{a}^{\prime} I^{\prime}}^{\dot{b}^{\prime}} \\
& =\bar{k}^{\nu_{R}} \delta_{\dot{a}}^{\dot{1}} \delta_{1^{\prime}}^{J^{\prime}} \bar{\Delta}^{\dot{b}} \quad I \\
& \equiv \bar{k}^{\nu_{R}} \delta_{\dot{a}}^{\dot{1}} \delta_{1^{\prime}}^{J^{\prime}} \Delta_{\dot{b} I}
\end{aligned}
$$

We now evaluate

$$
\left(A_{(2)}\right)_{M}^{N}=\sum a_{M}^{P}\left[J A_{(1)} J^{-1}, b\right]_{P}^{N}
$$


First we have

$$
\begin{aligned}
\left(A_{(2)}\right)_{\alpha I}^{\beta J} & =\sum a_{\alpha I}^{\gamma K}\left(\left(J A_{(1)} J^{-1}\right)_{\gamma K}^{\delta L} b_{\delta L}^{\beta J}-b_{\gamma K}^{\delta L}\left(J A_{(1)} J^{-1}\right)_{\delta L}^{\beta J}\right) \\
& =\sum X_{\alpha}^{\prime \gamma}\left(\left(J A_{(1)} J^{-1}\right)_{\gamma I}^{\delta J} X_{\delta}^{\beta}-X_{\gamma}^{\delta}\left(J A_{(1)} J^{-1}\right)_{\delta I}^{\beta J}\right)
\end{aligned}
$$

Thus

$$
\begin{aligned}
& \left(A_{(2)}\right)_{\dot{a} I}^{b J}=\sum X_{\dot{a}}^{\prime \dot{c}}\left(\left(J A_{(1)} J^{-1}\right)_{\dot{c} I}^{d J} X_{d}^{b}-X_{\dot{c}}^{\dot{d}}\left(J A_{(1)} J^{-1}\right)_{\dot{d} I}^{b J}\right) \\
& =\sum X_{\dot{a}}^{\prime} \dot{c}\left(\left(\left(k^{\nu}-k^{u}\right) \delta_{\dot{c}}^{\dot{1}} \delta_{1}^{d}+\left(k^{e}-k^{d}\right) \delta_{\dot{c}}^{\dot{2}} \delta_{2}^{d}\right) X_{d}^{b}\right. \\
& \left.-X_{\dot{c}}^{\dot{d}}\left(\left(k^{\nu}-k^{u}\right) \delta_{\dot{d}}^{\dot{1}} \delta_{1}^{b}+\left(k^{e}-k^{d}\right) \delta_{\dot{d}}^{\dot{2}} \delta_{2}^{b}\right)\right) \Sigma_{I}^{J t} \\
& =\left(\left(k^{\nu}-k^{u}\right)\left(\sum X_{\dot{a}}^{\prime \dot{i}} X_{1}^{b}-X_{\dot{a}}^{\prime} \dot{c} X_{\dot{c}}^{\dot{1}} \delta_{1}^{b}\right)+\left(k^{e}-k^{d}\right)\left(\sum X_{\dot{a}}^{\prime \dot{2}} X_{2}^{b}-X_{\dot{a}}^{\prime} X_{\dot{c}}^{\dot{2}} \delta_{2}^{b}\right)\right) \Sigma_{I}^{J t} \\
& =\left(\left(k^{\nu}-k^{u}\right) \phi_{\dot{a}}^{b}+\left(k^{e}-k^{d}\right) \widetilde{\phi}_{\dot{a}}^{b}\right) \Sigma_{I}^{J t}
\end{aligned}
$$

From the above calculation it should be clear that $\left(A_{(2)}\right)_{\dot{a} I}^{b J}$ could be expressed in terms of the fundamental Higgs fields $\phi_{\dot{a}}^{b}$ and $\Sigma_{I}^{J}$ as a consequence of the special form of the initial Dirac operator which satisfies the order one condition for the subalgebra (3.2). If this was not the case, then the field $\left(A_{(2)}\right)_{\dot{a} I}^{b J}$ would be an independent and thus fundamental Higgs field. Similarly $\left(A_{(2)}\right)_{a I}^{\dot{b} J}$ is the Hermitian conjugate of $\left(A_{(2)}\right)_{\dot{b} I}^{a J}$.. Next we have

$$
\begin{aligned}
\left(A_{(2)}\right)_{\dot{a} I}^{\dot{b}^{\prime} J^{\prime}} & =\sum X_{\dot{a}}^{\dot{c}}\left(\left(J A_{(1)} J^{-1}\right)_{\dot{c} I}^{\dot{b}^{\prime} L^{\prime}} Y_{L^{\prime}}^{J^{\prime}}-X_{\dot{c}}^{\dot{d}}\left(J A_{(1)} J^{-1}\right)_{\dot{d} I}^{\dot{b}^{\prime} J^{\prime}}\right) \\
& =\bar{k}^{\nu_{R}} \sum\left(X_{\dot{a}}^{\prime \dot{1}} Y_{1^{\prime}}^{J^{\prime}}-X_{\dot{a}}^{\prime \dot{c}} X_{\dot{c}}^{\dot{1}} \delta_{1^{\prime}}^{J^{\prime}}\right) \bar{\Delta}_{I}^{\dot{b}}{ }_{I} \\
& =\bar{k}^{\nu_{R}} \Delta_{\dot{a}} J^{\prime} \bar{\Delta}_{I}^{\dot{b}} \\
& =k^{* \nu_{R}} \Delta_{\dot{a} J} \Delta_{\dot{b} I}
\end{aligned}
$$

Collecting all terms we get

$$
\begin{aligned}
\left(D_{A}\right)_{\dot{a} I}^{b J}= & \left(\delta_{\dot{a}}^{\dot{1}} \delta_{1}^{b} k^{\nu}+\delta_{\dot{a}}^{2} \delta_{2}^{b} k^{e}\right) \delta_{I}^{1} \delta_{1}^{J}+\left(\delta_{\dot{a}}^{\dot{1}} \delta_{1}^{b} k^{u}+\delta_{\dot{a}}^{2} \delta_{2}^{b} k^{d}\right) \delta_{I}^{i} \delta_{j}^{J} \delta_{i}^{j} \\
& +\delta_{I}^{1} \delta_{1}^{J}\left(k^{\nu} \phi_{\dot{a}}^{b}+k^{e} \widetilde{\phi}_{\dot{a}}^{b}\right)+\delta_{I}^{i} \delta_{j}^{J} \delta_{i}^{j}\left(k^{u} \phi_{\dot{a}}^{b}+k^{d} \widetilde{\phi}_{\dot{a}}^{b}\right) \\
& +\left(\left(k^{\nu}-k^{u}\right) \delta_{\dot{a}}^{\dot{1}} \delta_{1}^{b}+\left(k^{e}-k^{d}\right) \delta_{\dot{a}}^{2} \delta_{2}^{b}\right) \Sigma_{I}^{J t} \\
& +\left(\left(k^{\nu}-k^{u}\right) \phi_{\dot{a}}^{b}+\left(k^{e}-k^{d}\right) \widetilde{\phi}_{\dot{a}}^{b}\right) \Sigma_{I}^{J t} \\
& =\left(k^{\nu}\left(\delta_{\dot{a}}^{\dot{1}} \delta_{1}^{b}+\phi_{\dot{a}}^{b}\right)+k^{e}\left(\delta_{\dot{a}}^{2} \delta_{2}^{b}+\widetilde{\phi}_{\dot{a}}^{b}\right)\right)\left(\delta_{I}^{1} \delta_{1}^{J}+\Sigma_{I}^{J t}\right) \\
& +\left(k^{u}\left(\delta_{\dot{a}}^{\dot{1}} \delta_{1}^{b}+\phi_{\dot{a}}^{b}\right)+k^{d}\left(\delta_{\dot{a}}^{2} \delta_{2}^{b}+\widetilde{\phi}_{\dot{a}}^{b}\right)\right)\left(\delta_{I}^{i} \delta_{j}^{J} \delta_{i}^{j}-\Sigma_{I}^{J t}\right)
\end{aligned}
$$

The other non-vanishing term is

$$
\begin{aligned}
\left(D_{A}\right)_{\dot{a} I}^{{ }^{b^{\prime}} J^{\prime}} & =k^{* \nu_{R}}\left(\delta_{\dot{a}}^{\dot{1}} \delta_{\dot{1}^{\prime}}^{\dot{b}^{\prime}} \delta_{I}^{1} \delta_{1^{\prime}}^{J^{\prime}}+\delta_{\dot{1}}^{\dot{b}} \delta_{I}^{1} \Delta_{\dot{a}}{ }^{J^{\prime}}+\delta_{\dot{a}}^{\dot{1}} \delta_{1^{\prime}}^{J^{\prime}} \bar{\Delta}_{I}^{\dot{b}}{ }_{I}+\Delta_{\dot{a}}{ }^{J^{\prime}} \bar{\Delta}_{I}^{\dot{b}}{ }_{I}\right) \\
& =k^{* \nu_{R}}\left(\delta_{\dot{a}}^{\dot{1}} \delta_{1^{\prime}}^{J^{\prime}}+\Delta_{\dot{a}}{ }^{J^{\prime}}\right)\left(\delta_{\dot{1}}^{\dot{b}} \delta_{I}^{1}+\bar{\Delta}_{I}^{\dot{b}}{ }_{I}\right)
\end{aligned}
$$




$$
\begin{aligned}
& \equiv k^{* \nu_{R}}\left(\delta_{\dot{a}}^{\dot{1}} \delta_{J}^{1}+\Delta_{\dot{a} J}\right)\left(\delta_{\dot{b}}^{\dot{1}} \delta_{I}^{1}+\Delta_{\dot{b} I}\right) \\
& \equiv\left(D_{A}\right)_{\dot{a} I \dot{b} J}
\end{aligned}
$$

All other non-vanishing terms are related to the above two by Hermitian conjugation.

Note that $D_{\dot{a} I}^{b J}$ gives, after spontaneous breaking, the Dirac masses while $D_{\dot{a} I}^{\dot{b}^{\prime} J^{\prime}}$ gives the Majorana masses. The Higgs fields are composite, the fundamental ones being of similar form to those of the fermion bilinear.

It is possible to absorb the constant terms (vacuum expectation values) by redefining the fields

$$
\begin{aligned}
\delta_{\dot{a}}^{\dot{1}} \delta_{1}^{b}+\phi_{\dot{a}}^{b} & \rightarrow \phi_{\dot{a}}^{b} \\
\delta_{\dot{a}}^{1} \delta_{J}^{1}+\Delta_{\dot{a} J} & \rightarrow \Delta_{\dot{a} J} \\
\delta_{I}^{1} \delta_{1}^{J}+\Sigma_{I}^{J t} & \rightarrow \Sigma_{I}^{J}
\end{aligned}
$$

so that when the potential of the spectral action is minimized one will get

$$
\begin{aligned}
\left\langle\phi_{\dot{a}}^{b}\right\rangle & =\delta_{\dot{a}}^{\dot{1}} \delta_{1}^{b} \\
\left\langle\Delta_{\dot{a} J}\right\rangle & =\delta_{\dot{a}}^{\dot{1}} \delta_{J}^{1} \\
\left\langle\Sigma_{I}^{J}\right\rangle & =\delta_{I}^{1} \delta_{1}^{J}
\end{aligned}
$$

Thus

$$
\begin{aligned}
\left(D_{A}\right)_{\dot{a} I}^{b J} & =\gamma_{5}\left(\left(k^{\nu} \phi_{\dot{a}}^{b}+k^{e} \widetilde{\phi}_{\dot{a}}^{b}\right) \Sigma_{I}^{J}+\left(k^{u} \phi_{\dot{a}}^{b}+k^{d} \widetilde{\phi}_{\dot{a}}^{b}\right)\left(\delta_{I}^{J}-\Sigma_{I}^{J}\right)\right) \equiv \gamma_{5} \Sigma_{\dot{a} I}^{b J} \\
\left(D_{A}\right)_{\dot{a} I}^{\dot{b}^{\prime} J^{\prime}} & =\gamma_{5} k^{* \nu_{R}} \Delta_{\dot{a} J} \Delta_{\dot{b} I} \equiv \gamma_{5} H_{\dot{a} I \dot{b} J}
\end{aligned}
$$

and the fundamental Higgs fields are $\left(2_{R}, 2_{L}, 1\right),\left(2_{R}, 1_{L}, 4\right),\left(1_{R}, 1_{L}, 1+15\right)$. The last of which $\Sigma_{I}^{J}$ drops out in the case when we take the lepton and quark Yukawa couplings to be identical. This is a realistic possibility and has the advantage that the Higgs sector becomes minimal. If, however, we start with a generic initial Dirac operator, then the fields $\Sigma_{\dot{a} I}^{b J}$ and $H_{\dot{a} I \dot{b} J}$ will be independent fundamental fields in the $\left(2_{R}, 2_{L}, 1+15\right)$ and $\left(3_{R}, 1_{L}, 10\right)$ and $\left(1_{R}, 1_{L}, 6\right)$ representations of $\mathrm{SU}(2)_{R} \times \mathrm{SU}(2)_{L} \times \mathrm{SU}(4)$.

The full Dirac operator on the product space $M \times F$ is

$$
\left(D_{A}\right)=\gamma^{\mu} D_{\mu} \otimes 1+\gamma_{5} D_{F}
$$

This gives the gauge fields

$$
A_{\alpha I}^{\beta J}=\gamma^{\mu} \sum X_{\alpha}^{\prime \gamma} \partial_{\mu} X_{\gamma}^{\beta} \delta_{I}^{J}
$$

and in particular

$$
\begin{aligned}
A_{\dot{a} I}^{\dot{b} J} & =\gamma^{\mu} \sum X_{\dot{a}}^{i \dot{c}} \partial_{\mu} X_{\dot{c}}^{\dot{b}} \delta_{I}^{J} \\
& =\gamma^{\mu}\left(-\frac{i}{2} g_{R} W_{\mu R}^{\alpha}\right)\left(\sigma^{\alpha}\right)_{\dot{a}}^{\dot{b}} \delta_{I}^{J}
\end{aligned}
$$


which is the gauge field of $\mathrm{SU}(2)_{R}$. Notice that $W_{\mu R}^{\alpha}$ are $\mathrm{SU}(2)_{R}$ and not $U(2)$ gauge fields because $X_{\dot{a}}^{i c} \partial_{\mu} X_{\dot{c}}^{\dot{b}}$ depend on quaternionic elements. Similarly

$$
\begin{aligned}
A_{a I}^{b J} & =\gamma^{\mu} \sum X_{a}^{\prime c} \partial_{\mu} X_{c}^{b} \delta_{I}^{J} \\
& =\gamma^{\mu}\left(-\frac{i}{2} g_{L} W_{\mu L}^{\alpha}\right)\left(\sigma^{\alpha}\right)_{a}^{b} \delta_{I}^{J}
\end{aligned}
$$

where the $W_{\mu L}^{\alpha}$ are $\mathrm{SU}(2)_{L}$ gauge fields. In the conjugate sector we have

$$
\begin{aligned}
A_{\alpha^{\prime} I^{\prime}}^{\beta^{\prime} J^{\prime}} & =\gamma^{\mu} \delta_{\alpha^{\prime}}^{\beta^{\prime}} \sum Y_{I^{\prime}}^{\prime K^{\prime}} \partial_{\mu} Y_{K^{\prime}}^{J^{\prime}} \\
& =\gamma^{\mu} \delta_{\alpha^{\prime}}^{\beta^{\prime}}\left(\frac{i}{2} g V_{\mu}^{m}\left(\lambda^{m}\right)_{I^{\prime}}^{J^{\prime}}+\frac{i}{2} g V_{\mu} \delta_{I^{\prime}}^{J^{\prime}}\right)
\end{aligned}
$$

where $V_{\mu}^{m}$ and $V_{\mu}$ are the $U(4)$ gauge fields. This implies that

$$
\begin{aligned}
\left(J A J^{-1}\right)_{\dot{a} I}^{\dot{b} J} & =-\gamma^{\mu} \delta_{\dot{a}}^{\dot{b}}\left(\frac{i}{2} g V_{\mu}^{m}\left(\lambda^{m}\right)_{I}^{J}+\frac{i}{2} g V_{\mu} \delta_{I}^{J}\right) \\
\left(J A J^{-1}\right)_{a I}^{b J} & =-\gamma^{\mu} \delta_{a}^{b}\left(\frac{i}{2} g V_{\mu}^{m}\left(\lambda^{m}\right)_{I}^{J}+\frac{i}{2} g V_{\mu} \delta_{I}^{J}\right)
\end{aligned}
$$

where

$$
\operatorname{Tr}\left(\lambda^{m}\right)=0
$$

are the generators of the group SU (4). We deduce that we get new contributions to

$$
\begin{aligned}
& \left(D_{A}\right)_{\dot{a} I}^{\dot{b} J}=\gamma^{\mu}\left(D_{\mu} \delta_{\dot{a}}^{\dot{b}} \delta_{I}^{J}-\frac{i}{2} g_{R} W_{\mu R}^{\alpha}\left(\sigma^{\alpha}\right)_{\dot{a}}^{\dot{b}} \delta_{I}^{J}-\delta_{\dot{a}}^{\dot{b}}\left(\frac{i}{2} g V_{\mu}^{m}\left(\lambda^{m}\right)_{I}^{J}+\frac{i}{2} g V_{\mu} \delta_{I}^{J}\right)\right) \\
& \left(D_{A}\right)_{a I}^{b J}=\gamma^{\mu}\left(D_{\mu} \delta_{a}^{b} \delta_{I}^{J}-\frac{i}{2} g_{L} W_{\mu L}^{\alpha}\left(\sigma^{\alpha}\right)_{a}^{b} \delta_{I}^{J}-\delta_{a}^{b}\left(\frac{i}{2} g V_{\mu}^{m}\left(\lambda^{m}\right)_{I}^{J}+\frac{i}{2} g V_{\mu} \delta_{I}^{J}\right)\right)
\end{aligned}
$$

The requirement that $A$ is unimodular implies that

$$
\operatorname{Tr}(A)=0
$$

which gives the condition

$$
V_{\mu}=0
$$

and thus the gauge group of this space is

$$
\mathrm{SU}(2)_{R} \times \mathrm{SU}(2)_{L} \times \mathrm{SU}(4)
$$

Summarizing, we have

$$
\begin{aligned}
& \left(D_{A}\right)_{\dot{a} I}^{\dot{b} J}=\gamma^{\mu}\left(D_{\mu} \delta_{\dot{a}}^{\dot{b}} \delta_{I}^{J}-\frac{i}{2} g_{R} W_{\mu R}^{\alpha}\left(\sigma^{\alpha}\right)_{\dot{a}}^{\dot{b}} \delta_{I}^{J}-\delta_{\dot{a}}^{\dot{b}}\left(\frac{i}{2} g V_{\mu}^{m}\left(\lambda^{m}\right)_{I}^{J}\right)\right) \otimes 1_{3} \\
& \left(D_{A}\right)_{a I}^{b J}=\gamma^{\mu}\left(D_{\mu} \delta_{a}^{b} \delta_{I}^{J}-\frac{i}{2} g_{L} W_{\mu L}^{\alpha}\left(\sigma^{\alpha}\right)_{a}^{b} \delta_{I}^{J}-\delta_{a}^{b}\left(\frac{i}{2} g V_{\mu}^{m}\left(\lambda^{m}\right)_{I}^{J}\right)\right) \otimes 1_{3}
\end{aligned}
$$




$$
\begin{aligned}
\left(D_{A}\right)_{\dot{a} I}^{b J} & =\gamma_{5}\left(\left(k^{\nu} \phi_{\dot{a}}^{b}+k^{e} \widetilde{\phi}_{\dot{a}}^{b}\right) \Sigma_{I}^{J}+\left(k^{u} \phi_{\dot{a}}^{b}+k^{d} \widetilde{\phi}_{\dot{a}}^{b}\right)\left(\delta_{I}^{J}-\Sigma_{I}^{J}\right)\right) \equiv \gamma_{5} \Sigma_{\dot{a} I}^{b J} \\
\left(D_{A}\right)_{\dot{a} I}^{\dot{b}^{\prime} J^{\prime}} & =\gamma_{5} k^{* \nu_{R}} \Delta_{\dot{a} J} \Delta_{\dot{b} I} \equiv \gamma_{5} H_{\dot{a} I \dot{b} J}
\end{aligned}
$$

where $1_{3}$ is for generations and

$$
D_{\mu}=\partial_{\mu}+\frac{1}{4} \omega_{\mu}^{c d}(e) \gamma_{c d}
$$

and other components are related to the ones above by

$$
D_{A^{\prime}}^{B^{\prime}}=\bar{D}_{A}^{B}, \quad D_{A^{\prime}}^{B}=\bar{D}_{A}^{B^{\prime}}, \quad D_{A}^{B^{\prime}}=\bar{D}_{A^{\prime}}^{B}
$$

Again, for generic initial Dirac operators $\Sigma_{\dot{a} I}^{b J}$ and $H_{\dot{a} I \dot{b} J}$ will be independent fundamental fields.

We now proceed to calculate $\left(D_{A}\right)^{2}$. The first step is to expand $D^{2}$ into the form

$$
\left(D_{A}\right)^{2}=-\left(g^{\mu \nu} \partial_{\mu} \partial_{\nu}+\mathcal{A}^{\mu} \partial_{\mu}+B\right)
$$

and from this extract the connection $\omega_{\mu}$

$$
\left(D_{A}\right)^{2}=-\left(g^{\mu \nu} \nabla_{\mu} \nabla_{\nu}+E\right)
$$

where

$$
\nabla_{\mu}=\partial_{\mu}+\omega_{\mu}
$$

This gives

$$
\begin{aligned}
\omega_{\mu} & =\frac{1}{2} g_{\mu \nu}\left(\mathcal{A}^{\nu}+\Gamma^{\nu}\right) \\
E & =B-g^{\mu \nu}\left(\partial_{\mu} \omega_{\nu}+\omega_{\mu} \omega_{\nu}-\Gamma_{\mu \nu}^{\rho} \omega_{\rho}\right) \\
\Omega_{\mu \nu} & =\partial_{\mu} \omega_{\nu}-\partial_{\nu} \omega_{\mu}+\left[\omega_{\mu}, \omega_{\nu}\right]
\end{aligned}
$$

where $\Gamma^{\nu}=g^{\rho \sigma} \Gamma_{\rho \sigma}^{\nu}$ and $\Gamma_{\mu \nu}^{\rho}$ is the Christoffel connection of the metric $g_{\mu \nu}$. We now proceed to evaluate the various components of $D^{2}$ :

$$
\begin{aligned}
\left(\left(D_{A}\right)^{2}\right)_{a I}^{b J}= & \left(D_{A}\right)_{a I}^{\dot{c} K}\left(D_{A}\right)_{\dot{c} K}^{b J}+\left(D_{A}\right)_{a I}^{c K}\left(D_{A}\right)_{c K}^{b J} \\
= & \Sigma_{a I}^{\dot{c} K} \sum_{\dot{c} K}^{b J} \\
& \quad+\left[\gamma^{\mu}\left(D_{\mu} \delta_{a}^{c} \delta_{I}^{K}-\frac{i}{2} g_{L} W_{\mu L}^{\alpha}\left(\sigma^{\alpha}\right)_{a}^{c} \delta_{I}^{K}+\delta_{a}^{c}\left(\frac{i}{2} g V_{\mu}^{m}\left(\lambda^{m}\right)_{I}^{K}\right)\right)\right. \\
& \left.\quad \cdot \gamma^{\nu}\left(D_{\nu} \delta_{c}^{b} \delta_{K}^{J}-\frac{i}{2} g_{L} W_{\nu L}^{\alpha}\left(\sigma^{\alpha}\right)_{c}^{b} \delta_{K}^{J}+\delta_{c}^{b}\left(\frac{i}{2} g V_{\mu}^{m}\left(\lambda^{m}\right)_{K}^{J}\right)\right)\right] 1_{3} \\
\left(\left(D_{A}\right)^{2}\right)_{\dot{a} I}^{\dot{b} J}= & \left(D_{A}\right)_{\dot{a} I}^{\dot{c} K}\left(D_{A}\right)_{\dot{c} K}^{\dot{b} J}+\left(D_{A}\right)_{\dot{a} I}^{c K}\left(D_{A}\right)_{c K}^{\dot{b} J}+\left(D_{A}\right)_{\dot{a} I}^{\dot{c} K^{\prime}}\left(D_{A}\right)_{\dot{c} K^{\prime} K^{\prime}}^{\dot{b} J} \\
= & H_{\dot{a} I \dot{c} K K} H^{\dot{c} K \dot{b} J}+\sum_{\dot{a} I}^{c K} \sum_{c K}^{\dot{b} J}
\end{aligned}
$$




$$
\begin{gathered}
+\left[\gamma^{\mu}\left(D_{\mu} \delta_{\dot{a}}^{\dot{c}} \delta_{I}^{K}-\frac{i}{2} g_{R} W_{\mu R}^{\alpha}\left(\sigma^{\alpha}\right)_{\dot{a}}^{\dot{c}} \delta_{I}^{K}+\delta_{\dot{a}}^{\dot{c}}\left(\frac{i}{2} g V_{\mu}^{m}\left(\lambda^{m}\right)_{I}^{K}\right)\right)\right. \\
\left.\cdot \gamma^{\nu}\left(D_{\nu} \delta_{\dot{c}}^{\dot{b}} \delta_{K}^{J}-\frac{i}{2} g_{R} W_{\nu R}^{\alpha}\left(\sigma^{\alpha}\right)_{\dot{c}}^{\dot{b}} \delta_{K}^{J}+\delta_{\dot{c}}^{\dot{b}}\left(\frac{i}{2} g V_{\mu}^{m}\left(\lambda^{m}\right)_{K}^{J}\right)\right)\right] \\
\left(\left(D_{A}\right)^{2}\right)_{\dot{a} I}^{b J}=\left(D_{A}\right)_{\dot{a} I}^{c K}\left(D_{A}\right)_{c K}^{b J}+\left(D_{A}\right)_{\dot{a} I}^{\dot{c} K}\left(D_{A}\right)_{\dot{c} K}^{b J} \\
=\gamma_{5} \gamma^{\mu} \Sigma_{\dot{a} I}^{c J}\left(D_{\mu} \delta_{c}^{b} \delta_{K}^{J}-\frac{i}{2} g_{L} W_{\mu L}^{\alpha}\left(\sigma^{\alpha}\right)_{c}^{b} \delta_{K}^{J}+\delta_{c}^{b}\left(\frac{i}{2} g V_{\mu}^{m}\left(\lambda^{m}\right)_{K}^{J}\right)\right) \\
-\gamma_{5} \gamma^{\mu}\left(D_{\mu} \delta_{\dot{a}}^{\dot{c}} \delta_{I}^{K}-\frac{i}{2} g_{R} W_{\mu R}^{\alpha}\left(\sigma^{\alpha}\right)_{\dot{a}}^{\dot{c}} \delta_{I}^{K}+\delta_{\dot{a}}^{\dot{c}}\left(\frac{i}{2} g V_{\mu}^{m}\left(\lambda^{m}\right)_{I}^{K}\right)\right) \Sigma_{\dot{c} K}^{b J} \\
=\gamma^{\mu} \gamma_{5} \nabla_{\mu} \Sigma_{\dot{a} I}^{b J}
\end{gathered}
$$

where the covariant derivative $\nabla_{\mu}$ is with respect to the gauge group $\mathrm{SU}(2)_{R} \times \mathrm{SU}(2)_{L} \times$ $\mathrm{SU}(4)$.

$$
\begin{aligned}
\left(\left(D_{A}\right)^{2}\right)_{\dot{a} I}^{\dot{b}^{\prime} J^{\prime}} & =\left(D_{A}\right)_{\dot{a} I}^{\dot{c} K}\left(D_{A}\right)_{\dot{c} K}^{\dot{b}^{\prime} J^{\prime}}+\left(D_{A}\right)_{\dot{a} I}^{c^{\prime} K^{\prime}}\left(D_{A}\right)_{\dot{c}^{\prime} K^{\prime}}^{\dot{b}^{\prime} J^{\prime}} \\
& =\gamma^{\mu} \gamma_{5}\left(D_{\mu} \delta_{\dot{a}}^{\dot{c}} \delta_{I}^{K}-\frac{i}{2} g_{R} W_{\mu R}^{\alpha}\left(\sigma^{\alpha}\right)_{\dot{a}}^{\dot{c}} \delta_{I}^{K}+\delta_{\dot{a}}^{\dot{c}}\left(\frac{i}{2} g V_{\mu}^{m}\left(\lambda^{m}\right)_{I}^{K}\right)\right) H_{\dot{c} K \dot{b} J} \\
& -\gamma^{\mu} \gamma_{5} H_{\dot{a} I \dot{c} K}\left(D_{\mu} \delta_{\dot{c}}^{\dot{b}} \delta_{K}^{J}-\frac{i}{2} g_{R} W_{\mu R}^{\alpha}\left(\sigma^{\alpha}\right)_{\dot{c}}^{\dot{b}} \delta_{K}^{J}+\delta_{\dot{c}}^{\dot{b}}\left(\frac{i}{2} g V_{\mu}^{m}\left(\lambda^{m}\right)_{K}^{J}\right)\right) \\
& =\gamma^{\mu} \gamma_{5} \nabla_{\mu} H_{\dot{a} I \dot{b} J}
\end{aligned}
$$

where the covariant derivative now will be with respect to $\mathrm{SU}(2)_{R} \times \mathrm{SU}(4)$. Next we have

$$
\begin{aligned}
\left(D^{2}\right)_{\dot{a} I}^{b^{\prime} J^{\prime}} & =D_{\dot{a} I}^{\dot{c}^{\prime} K^{\prime}} D_{\dot{c}^{\prime} K^{\prime}}^{b^{\prime} J^{\prime}} \\
& =H_{\dot{a} I \dot{c} K} \bar{\Sigma}_{b J}^{\dot{c} K}
\end{aligned}
$$

and finally

$$
\begin{aligned}
\left(\left(D_{A}\right)^{2}\right)_{a I}^{\dot{b}^{\prime} J^{\prime}} & =\left(D_{A}\right)_{\dot{a} I}^{\dot{c} K}\left(D_{A}\right)_{\dot{c} K}^{\dot{b}^{\prime} J^{\prime}} \\
& =\Sigma_{\dot{a} I}^{\dot{c} K} H_{\dot{c} K \dot{b} J}
\end{aligned}
$$

We then list the entries of the matrices $\left(\omega_{\mu}\right)_{M}^{N},(E)_{M}^{N}$ which are deduced from the form of the operator $\left(D_{A}\right)^{2}$. First we have

$$
\begin{aligned}
\left(\omega_{\mu}\right)_{a I}^{b J} & =\left(\left(\frac{1}{4} \omega_{\mu}^{c d}(e) \gamma_{c d}\right) \delta_{a}^{b} \delta_{I}^{J}-\frac{i}{2} g_{L} W_{\mu L}^{\alpha}\left(\sigma^{\alpha}\right)_{a}^{b} \delta_{I}^{J}-\frac{i}{2} g V_{\mu}^{m}\left(\lambda^{m}\right)_{I}^{J} \delta_{a}^{b}\right) \otimes 1_{3} \\
\left(\omega_{\mu}\right)_{\dot{a} I}^{\dot{b} J} & =\left(\left(\frac{1}{4} \omega_{\mu}^{c d}(e) \gamma_{c d}\right) \delta_{\dot{a}}^{\dot{b}} \delta_{I}^{J}-\frac{i}{2} g_{R} W_{\mu L}^{\alpha}\left(\sigma^{\alpha}\right)_{\dot{a}}^{\dot{b}} \delta_{I}^{J}-\frac{i}{2} g \delta_{\dot{a}}^{b} V_{\mu}^{m}\left(\lambda^{m}\right)_{I}^{J}\right) \otimes 1_{3} \\
\left(\omega_{\mu}\right)_{A^{\prime}}^{B^{\prime}} & =\left(\bar{\omega}_{\mu}\right)_{A}^{B}
\end{aligned}
$$

This in turn implies that the components of the curvature

$$
\Omega_{\mu \nu}=\partial_{\mu} \omega_{\nu}-\partial_{\nu} \omega_{\mu}+\left[\omega_{\mu}, \omega_{\nu}\right]
$$


are given by

$$
\begin{aligned}
\left(\Omega_{\mu \nu}\right)_{a I}^{b J} & =\left(\left(\frac{1}{4} R_{\mu \nu}^{c d} \gamma_{c d}\right) \delta_{a}^{b} \delta_{I}^{J}-\frac{i}{2} g_{L} W_{\mu \nu L}^{\alpha}\left(\sigma^{\alpha}\right)_{a}^{b} \delta_{I}^{J}-\frac{i}{2} g V_{\mu \nu}^{m}\left(\lambda^{m}\right)_{I}^{J} \delta_{a}^{b}\right) \otimes 1_{3} \\
\left(\Omega_{\mu \nu}\right)_{\dot{a} I}^{\dot{b} J} & =\left(\left(\frac{1}{4} R_{\mu \nu}^{c d} \gamma_{c d}\right) \delta_{\dot{a}}^{b} \delta_{I}^{J}-\frac{i}{2} g_{R} W_{\mu \nu R}^{\alpha}\left(\sigma^{\alpha}\right)_{\dot{a}}^{\dot{b}} \delta_{I}^{J}-\frac{i}{2} g V_{\mu \nu}^{m}\left(\lambda^{m}\right)_{I}^{J} \delta_{\dot{a}}^{\dot{b}}\right) \otimes 1_{3} \\
\left(\Omega_{\mu \nu}\right)_{A^{\prime}}^{B^{\prime}} & =\left(\bar{\Omega}_{\mu \nu}\right)_{A}^{B}
\end{aligned}
$$

Comparing with equation (A.61) we deduce that

$$
\begin{aligned}
&-(E)_{a I}^{b J}=\left(\left(\frac{1}{4} R \delta_{a}^{b} \delta_{I}^{J}+\frac{1}{2} \gamma^{\mu \nu}\left(-\frac{i}{2} g_{L} W_{\mu \nu L}^{\alpha}\left(\sigma^{\alpha}\right)_{a}^{b} \delta_{I}^{J}-\frac{i}{2} g V_{\mu \nu}^{m}\left(\lambda^{m}\right)_{I}^{J} \delta_{a}^{b}\right)\right) 1_{3}\right. \\
&\left.+\Sigma_{a I}^{\dot{c} K} \Sigma_{\dot{c} K}^{b J}\right) \\
&(-E)_{\dot{a} I}^{\dot{b} J}=\left(\left(\frac{1}{4} R \delta_{\dot{a}}^{\dot{b}} \delta_{I}^{J}+\frac{1}{2} \gamma^{\mu \nu}\left(-\frac{i}{2} g_{R} W_{\mu \nu R}^{\alpha}\left(\sigma^{\alpha}\right)_{\dot{a}}^{\dot{b}} \delta_{I}^{J}-\frac{i}{2} g V_{\mu \nu}^{m}\left(\lambda^{m}\right)_{I}^{J} \delta_{\dot{a}}^{\dot{b}}\right)\right) 1_{3}\right. \\
&\left.+H_{\dot{a} I \dot{c} K} H^{\dot{c} K K \dot{b} J}+\Sigma_{\dot{a} I}^{c K} \Sigma_{c K}^{\dot{b} J}\right) \\
&-(E)_{\dot{a} I}^{b J}=\gamma^{\mu} \gamma_{5} \nabla_{\mu} \Sigma_{\dot{a} I}^{b J} \\
&-(E)_{\dot{a} I}^{\dot{b}^{\prime} J^{\prime}}=\gamma^{\mu} \gamma_{5} \nabla_{\mu} H_{\dot{a} I \dot{b} J} \\
&(-E)_{\dot{a} I}^{b^{\prime} J^{\prime}}=H_{\dot{a} I \dot{c} K} \bar{\Sigma}_{b J}^{\dot{c} K} \\
&(-E)_{a I}^{\dot{b}^{\prime} J^{\prime}}=\Sigma_{a I}^{\dot{c} K} H_{\dot{c} K \dot{b} J}
\end{aligned}
$$

Evaluating the various traces of the $384 \times 384$ matrices on spinor and generation space, we get

$$
\begin{aligned}
\operatorname{Tr}(E) & =\operatorname{tr}\left(E_{A}^{A}+E_{A^{\prime}}^{A^{\prime}}\right)=\operatorname{tr}\left(E_{A}^{A}+\bar{E}_{A}^{A}\right) \\
-\operatorname{tr}(E)_{a I}^{a I} & =4\left[\frac{3}{4} R(2)(4)+H_{a I}^{c \dot{K}} H_{\dot{c} K}^{a I}\right] \\
-\operatorname{tr}(E)_{\dot{a} I}^{\dot{a} J} & =4\left[\frac{3}{4} R(2)(4)+H_{\dot{a} I \dot{c} K} H^{\dot{c} K \dot{a} I}+\Sigma_{\dot{a} I}^{c K} \Sigma_{c K}^{\dot{a} I}\right] \\
-\frac{1}{2} \operatorname{Tr}(E) & =4\left(12 R+H_{\dot{a} I \dot{c} K} H^{\dot{c} K \dot{a} I}+2 \Sigma_{\dot{a} I}^{c K} \Sigma_{c K}^{\dot{a} I}\right)
\end{aligned}
$$

Next

$$
\begin{aligned}
\operatorname{Tr}\left(\Omega_{\mu \nu}^{2}\right)_{M}^{M} & =2 \operatorname{Tr}\left(\Omega_{\mu \nu}^{2}\right)_{A}^{A} \\
& =2 \operatorname{Tr}\left(\left(\Omega_{\mu \nu}^{2}\right)_{\dot{a} I}^{\dot{a} I}+\left(\Omega_{\mu \nu}^{2}\right)_{a I}^{a I}\right) \\
\operatorname{Tr}\left(\Omega_{\mu \nu}^{2}\right)_{a I}^{a I} & =\operatorname{Tr}\left(\left(\left(\frac{1}{4} R_{\mu \nu}^{c d} \gamma_{c d}\right) \delta_{a}^{b} \delta_{I}^{J}-\frac{i}{2} g_{L} W_{\mu \nu L}^{\alpha}\left(\sigma^{\alpha}\right)_{a}^{b} \delta_{I}^{J}-\frac{i}{2} g V_{\mu \nu}^{m}\left(\lambda^{m}\right)_{i}^{j} \delta_{a}^{b}\right) \otimes 1_{3}\right)^{2} \\
& =4\left[-\frac{1}{8} R_{\mu \nu \rho \sigma}^{2}(4)(2)(3)-\frac{1}{4} g_{L}^{2}\left(W_{\mu \nu}^{\alpha}\right)^{2}(4)(2)(3)-\frac{1}{4} g^{2}\left(V_{\mu \nu}^{m}\right)^{2}(3)(2)(2)\right] \\
& =4\left[-3 R_{\mu \nu \rho \sigma}^{2}-6 g_{L}^{2}\left(W_{\mu \nu L}^{\alpha}\right)^{2}-3 g^{2}\left(V_{\mu \nu}^{m}\right)^{2}\right]
\end{aligned}
$$




$$
\begin{aligned}
\operatorname{Tr}\left(\Omega_{\mu \nu}^{2}\right)_{\dot{a} I}^{\dot{a} I} & =\operatorname{Tr}\left(\left(\left(\frac{1}{4} R_{\mu \nu}^{c d} \gamma_{c d}\right) \delta_{\dot{a}}^{\dot{b}} \delta_{I}^{J}-\frac{i}{2} g_{R} W_{\mu \nu R}^{\alpha}\left(\sigma^{\alpha}\right)_{\dot{a}}^{\dot{b}} \delta_{I}^{J}-\frac{i}{2} g V_{\mu \nu}^{m}\left(\lambda^{m}\right)_{I}^{J} \delta_{\dot{a}}^{\dot{b}}\right) \otimes 1_{3}\right)^{2} \\
& =4\left[-3 R_{\mu \nu \rho \sigma}^{2}-6 g_{R}^{2}\left(W_{\mu \nu R}^{\alpha}\right)^{2}-3 g^{2}\left(V_{\mu \nu}^{m}\right)^{2}\right]
\end{aligned}
$$

Therefore

$$
\frac{1}{2} \operatorname{Tr}\left(\Omega_{\mu \nu}^{2}\right)_{M}^{M}=24\left[-R_{\mu \nu \rho \sigma}^{2}-g_{L}^{2}\left(W_{\mu \nu L}^{\alpha}\right)^{2}-g_{R}^{2}\left(W_{\mu \nu R}^{\alpha}\right)^{2}-g^{2}\left(V_{\mu \nu}^{m}\right)^{2}\right]
$$

Next we compute

$$
\left(E^{2}\right)_{A}^{B}=E_{A}^{C} E_{C}^{B}+E_{A}^{C^{\prime}} E_{C^{\prime}}^{B}
$$

and listing the components of this matrix we get

$$
\begin{aligned}
& \left(E^{2}\right)_{a I}^{b J}=E_{a I}^{c K} E_{c K}^{b j}+E_{a I}^{\dot{c} K} E_{\dot{c} K}^{b J}+E_{a I}^{\dot{c}^{\prime} K^{\prime}} E_{\dot{c}^{\prime} K^{\prime}}^{b J} \\
& \left(E^{2}\right)_{\dot{a} I}^{\dot{b} J}=E_{\dot{a} I}^{\dot{c} K} E_{\dot{c} K}^{\dot{b} J}+E_{\dot{a} I}^{c K} E_{c K}^{\dot{b} J}+E_{\dot{a} I}^{c^{\prime} K^{\prime}} E_{c^{\prime} K^{\prime}}^{\dot{b} J}+E_{\dot{a} I}^{\dot{c}^{\prime} K^{\prime}} E_{\dot{c}^{\prime} K^{\prime}}^{\dot{b} J}
\end{aligned}
$$

Collecting terms and tracing we obtain for the right-handed components

$$
\begin{aligned}
& \operatorname{tr}\left(E^{2}\right)_{\dot{a} I}^{\dot{a} I}=\operatorname{tr}\left\{\left(\gamma^{\mu} \gamma_{5} \nabla_{\mu} \Sigma_{\dot{a} I}^{b J} \gamma^{\nu} \gamma_{5} \nabla_{\nu} \Sigma_{b J}^{\dot{a} I}\right)+\left(\gamma^{\mu} \gamma_{5} \nabla_{\mu} H_{\dot{a} I \dot{I} J J} \gamma^{\nu} \gamma_{5} \nabla_{\nu} H^{\dot{a} I \dot{b} J}\right)\right. \\
& +H_{\dot{a} I \dot{c} K} \sum_{b J}^{\dot{c} K} H^{\dot{a} I \dot{d} L} \sum_{\dot{d} L}^{b J}+\left(\left(\frac{1}{4} R \delta_{\dot{a}}^{\dot{b}} \delta_{I}^{J}+\frac{1}{2} \gamma^{\mu \nu}\left(-\frac{i}{2} g_{R} W_{\mu \nu R}^{\alpha}\left(\sigma^{\alpha}\right)_{\dot{a}}^{\dot{b}} \delta_{I}^{J}\right.\right.\right. \\
& \left.\left.\left.\left.-\frac{i}{2} g V_{\mu \nu}^{m}\left(\lambda^{m}\right)_{I}^{J} \delta_{\dot{a}}^{\dot{b}}\right)\right) 1_{3}+H_{\dot{a} I \dot{c} K} H^{\dot{c} K \dot{b} J}+\Sigma_{\dot{a} I}^{c K} \Sigma_{c K}^{\dot{b} J}\right)^{2}\right\} \\
& =4\left[\frac{1}{4}(-2)\left(-\frac{1}{4} g_{R}^{2}\left(W_{\mu \nu R}^{\alpha}\right)^{2}(2)(4)(3)-\frac{1}{4} g^{2}\left(V_{\mu \nu}^{m}\right)^{2}(2)(2)(3)\right)\right. \\
& +\frac{1}{16} R^{2}(2)(4)(3)+\frac{1}{2} R\left(H_{\dot{a} I \dot{c} K} H^{\dot{c} K \dot{a} I}+\Sigma_{\dot{a} I}^{c K} \Sigma_{c K}^{\dot{a} I}\right)+\nabla_{\mu} H_{\dot{a} I \dot{b} J} \nabla^{\mu} H^{\dot{a} I \dot{b} J} \\
& \left.+\nabla_{\mu} \Sigma_{\dot{a} I}^{b J} \nabla^{\mu} \Sigma_{b J}^{\dot{a} I}+H_{\dot{a} I \dot{c} K} \Sigma_{b J}^{\dot{c} K} H^{\dot{a} I \dot{d} L} \sum_{\dot{d} L}^{b J}+\left|H_{\dot{a} I \dot{c} K} H^{\dot{c} K \dot{b} J}+\Sigma_{\dot{a} I}^{c K} \Sigma_{c K}^{\dot{b} J}\right|^{2}\right] \\
& =4\left[\frac{3}{2}\left(2 g_{R}^{2}\left(W_{\mu \nu R}^{\alpha}\right)^{2}+g_{3}^{2}\left(V_{\mu \nu}^{m}\right)^{2}\right)+\frac{3}{2} R^{2}+\nabla_{\mu} H_{\dot{a} I \dot{I} b J} \nabla^{\mu} H^{\dot{a} I \dot{b} J}\right. \\
& +\nabla_{\mu} \Sigma_{\dot{a} I}^{b J} \nabla^{\mu} \Sigma_{b J}^{\dot{a} I}+\frac{1}{2} R\left(H_{\dot{a} I \dot{c} K} H^{\dot{c} K \dot{a} I}+\Sigma_{\dot{a} I}^{c K} \Sigma_{c K}^{\dot{a} I}\right)+H_{\dot{a} I \dot{c} K} \Sigma_{b J}^{\dot{c} K} H^{\dot{a} I \dot{d} L} \sum_{\dot{d} L}^{b J} \\
& \left.+\left|H_{\dot{a} I \dot{c} K} H^{\dot{c} K \dot{b} J}+\Sigma_{\dot{a} I}^{c K} \Sigma_{c K}^{\dot{b} J}\right|^{2}\right]
\end{aligned}
$$

and for the left-handed components

$$
\begin{gathered}
\operatorname{tr}\left(E^{2}\right)_{a I}^{a I}=\operatorname{tr}\left\{\left(\left(\frac{R}{4} \delta_{a}^{b} \delta_{I}^{J}+\frac{1}{2} \gamma^{\mu \nu}\left(-\frac{i}{2} g_{L} W_{\mu \nu L}^{\alpha}\left(\sigma^{\alpha}\right)_{a}^{b} \delta_{I}^{J}-\frac{i}{2} g V_{\mu \nu}^{m}\left(\lambda^{m}\right)_{I}^{J}\right) \delta_{a}^{b}\right) 1_{3}\right.\right. \\
\left.\left.+\Sigma_{a I}^{c K} \Sigma_{\dot{c} K}^{b J}\right)^{2}+\gamma^{\mu} \gamma_{5} \nabla_{\mu} \Sigma_{a I}^{\dot{c} K} \gamma^{\nu} \gamma_{5} \nabla_{\nu} \Sigma_{\dot{c} K}^{a I}+\left|\Sigma_{a I}^{\dot{c} K} H_{\dot{c} I \dot{b} J}\right|^{2}\right\} \\
=4\left[\frac{1}{4}(-2)\left(-\frac{1}{4} g_{L}^{2}\left(W_{\mu \nu L}^{\alpha}\right)^{2}(2)(4)(3)-\frac{1}{4} g_{3}^{2}\left(V_{\mu \nu}^{m}\right)^{2}(2)(2)(3)\right)\right. \\
+\frac{1}{16} R^{2}(2)(4)(3)+\frac{1}{2} R \Sigma_{a I}^{\dot{c} K} \Sigma_{\dot{c} K}^{a I}+\nabla_{\mu} \Sigma_{a I}^{\dot{c} K} \nabla^{\mu} \Sigma_{\dot{c} K}^{a I} \\
\left.+\Sigma_{a I}^{\dot{c} K} \Sigma_{\dot{c} K}^{b J} \Sigma_{b J}^{\dot{d} L} \Sigma_{\dot{d} L}^{a I}+\left|\Sigma_{a I}^{\dot{c} K} H_{\dot{c} I \dot{b} J}\right|^{2}\right]
\end{gathered}
$$




$$
\begin{aligned}
=4\left[\frac { 3 } { 2 } \left(2 g_{L}^{2}(\right.\right. & \left.\left.W_{\mu \nu L}^{\alpha}\right)^{2}+g_{3}^{2}\left(V_{\mu \nu}^{m}\right)^{2}\right)+\frac{3}{2} R^{2}+\nabla_{\mu} \Sigma_{a I}^{\dot{c} K} \nabla^{\mu} \Sigma_{\dot{c} K}^{a I} \\
& \left.+\frac{1}{2} R \Sigma_{a I}^{\dot{c} K} \Sigma_{\dot{c} K}^{a I}+\Sigma_{a I}^{\dot{c} K} \Sigma_{\dot{c} K}^{b J} \Sigma_{b J}^{\dot{d} L} \Sigma_{\dot{d} L}^{a I}+\left|\Sigma_{a I}^{\dot{c} K} H_{\dot{c} I \dot{b} J}\right|^{2}\right]
\end{aligned}
$$

Collecting all terms we finally get

$$
\begin{aligned}
\frac{1}{2} \operatorname{tr}\left(E^{2}\right)=4[ & 3\left(g_{L}^{2}\left(W_{\mu \nu L}^{\alpha}\right)^{2}+g^{2}\left(V_{\mu \nu}^{m}\right)^{2}+g_{R}^{2}\left(W_{\mu \nu R}^{\alpha}\right)^{2}+R^{2}\right) \\
& +2 \nabla_{\mu} \Sigma_{a I}^{\dot{c} K} \nabla^{\mu} \Sigma_{\dot{c} K}^{a I}+\nabla_{\mu} H_{\dot{a} I \dot{b} J} \nabla^{\mu} H^{\dot{a} I \dot{b} J}+\frac{1}{2} R\left(H_{\dot{a} I \dot{c} K} H^{\dot{c} K \dot{a} I}+2 \Sigma_{\dot{a} I}^{c K} \sum_{c K}^{\dot{a} I}\right) \\
& \left.+2 \Sigma_{a I}^{\dot{c} K} \sum_{\dot{c} K}^{b J} \Sigma_{b J}^{\dot{d} L} \Sigma_{\dot{d} L}^{a I}+4 H_{\dot{a} I \dot{c} K} \Sigma_{b J}^{\dot{c} K} H^{\dot{a} I \dot{d} L} \sum_{\dot{d} L}^{b J}+\left|H_{\dot{a} I \dot{c} K} H^{\dot{c} K \dot{b} J}\right|^{2}\right]
\end{aligned}
$$

The first two Seely-de Witt coefficients are, first for $a_{0}$

$$
\begin{aligned}
a_{0} & =\frac{1}{16 \pi^{2}} \int d^{4} x \sqrt{g} \operatorname{Tr}(1) \\
& =\frac{1}{16 \pi^{2}}(4)(32)(3) \int d^{4} x \sqrt{g} \\
& =\frac{24}{\pi^{2}} \int d^{4} x \sqrt{g}
\end{aligned}
$$

then for $a_{2}$ :

$$
\begin{aligned}
a_{2} & =\frac{1}{16 \pi^{2}} \int d^{4} x \sqrt{g} \operatorname{Tr}\left(E+\frac{1}{6} R\right) \\
& =\frac{1}{16 \pi^{2}} \int d^{4} x \sqrt{g}\left(\left(R(-96+64)-8\left(H_{\dot{a} I \dot{c} K} H^{\dot{c} K \dot{a} I}+2 \Sigma_{\dot{a} I}^{c K} \sum_{c K}^{\dot{a} I}\right)\right)\right. \\
& =-\frac{2}{\pi^{2}} \int d^{4} x \sqrt{g}\left(R+\frac{1}{4}\left(H_{\dot{a} I \dot{c} K} H^{\dot{c} K \dot{a} I}+2 \Sigma_{\dot{a} I}^{c K} \sum_{c K}^{\dot{a} I}\right)\right)
\end{aligned}
$$

With all the above information we can now compute the Seeley-de Witt coefficient $a_{4}$ :

$$
a_{4}=\frac{1}{16 \pi^{2}} \int d^{4} x \sqrt{g} \operatorname{Tr}\left(\frac{1}{360}\left(5 R^{2}-2 R_{\mu \nu}^{2}+2 R_{\mu \nu \rho \sigma}^{2}\right) 1+\frac{1}{2}\left(E^{2}+\frac{1}{3} R E+\frac{1}{6} \Omega_{\mu \nu}^{2}\right)\right)
$$

and where we have omitted the surface terms. Thus

$$
\begin{aligned}
& \frac{1}{2} \operatorname{Tr}\left(E^{2}+\right.\left.\frac{1}{3} R E+\frac{1}{6} \Omega_{\mu \nu}^{2}\right) \\
&=4\left[3\left(g_{L}^{2}\left(W_{\mu \nu L}^{\alpha}\right)^{2}+g^{2}\left(V_{\mu \nu}^{m}\right)^{2}+g_{R}^{2}\left(W_{\mu \nu R}^{\alpha}\right)^{2}+R^{2}\right)+2 \nabla_{\mu} \Sigma_{a I}^{\dot{c} K} \nabla^{\mu} \Sigma_{\dot{c} K}^{a I}\right. \\
&+\nabla_{\mu} H_{\dot{a} I \dot{b} J} \nabla^{\mu} H^{\dot{a} I \dot{b} J}+\frac{1}{2} R\left(H_{\dot{a} I \dot{c} K} H^{\dot{c} K \dot{a} I}+2 \Sigma_{\dot{a} I}^{c K} \Sigma_{c K}^{\dot{a} I}\right) \\
&+4 H_{\dot{a} I \dot{c} K} \Sigma_{b J}^{\dot{c} K} H^{\dot{a} I \dot{d} L} \sum_{\dot{d} L}^{b J}+2 \Sigma_{a I}^{\dot{c} K} \Sigma_{\dot{c} K}^{b J} \Sigma_{b J}^{\dot{d L}} \Sigma_{\dot{d} L}^{a I}+\left|H_{\dot{a} I \dot{c} K} H^{\dot{c} K \dot{b} J}\right|^{2} \\
& \quad-\frac{1}{3} R\left(12 R+H_{\dot{a} I \dot{c} K} H^{\dot{c} K \dot{a} I}+2 \Sigma_{\dot{a} I}^{c K} \Sigma_{c K}^{\dot{a} I}\right) \\
&\left.-R_{\mu \nu \rho \sigma}^{2}-g_{L}^{2}\left(W_{\mu \nu L}^{\alpha}\right)^{2}-g_{R}^{2}\left(W_{\mu \nu R}^{\alpha}\right)^{2}-g^{2}\left(V_{\mu \nu}^{m}\right)^{2}\right]
\end{aligned}
$$




$$
\begin{aligned}
=4\left[-R_{\mu \nu \rho \sigma}^{2}\right. & -R^{2}+2 g_{L}^{2}\left(W_{\mu \nu L}^{\alpha}\right)^{2}+2 g_{R}^{2}\left(W_{\mu \nu R}^{\alpha}\right)^{2}+2 g^{2}\left(V_{\mu \nu}^{m}\right)^{2} \\
& +2 \nabla_{\mu} \Sigma_{a I}^{\dot{c} K} \nabla^{\mu} \Sigma_{\dot{c} K}^{a I}+\nabla_{\mu} H_{\dot{a} I \dot{b} J} \nabla^{\mu} H^{\dot{a} I \dot{I} J}+\frac{1}{6} R\left(H_{\dot{a} I \dot{c} K} H^{\dot{c} K \dot{a} I}+2 \Sigma_{\dot{a} I}^{c K} \Sigma_{c K}^{\dot{a} I}\right) \\
& \left.+\left|H_{\dot{a} I \dot{c} K} H^{\dot{c} K \dot{b} J}\right|^{2}+4 H_{\dot{a} I \dot{c} K} \Sigma_{b J}^{\dot{c} K} H^{\dot{a} I \dot{d} L} \sum_{\dot{d} L}^{b J}+2 \Sigma_{a I}^{\dot{c} K} \Sigma_{\dot{c} K}^{b J} \Sigma_{b J}^{\dot{d L}} \Sigma_{\dot{d} L}^{a I}\right]
\end{aligned}
$$

Collecting terms we get

$$
\begin{aligned}
a_{4}=\frac{1}{2 \pi^{2}} \int d^{4} x \sqrt{g}[ & \frac{1}{30}\left(5 R^{2}-8 R_{\mu \nu}^{2}-7 R_{\mu \nu \rho \sigma}^{2}\right)+g_{L}^{2}\left(W_{\mu \nu L}^{\alpha}\right)^{2} \\
& +g_{R}^{2}\left(W_{\mu \nu R}^{\alpha}\right)^{2}+g^{2}\left(V_{\mu \nu}^{m}\right)^{2}+\nabla_{\mu} \Sigma_{a I}^{\dot{c} K} \nabla^{\mu} \sum_{\dot{c} K}^{a I}+\frac{1}{2} \nabla_{\mu} H_{\dot{a} I \dot{b} J} \nabla^{\mu} H^{\dot{a} I \dot{b} J} \\
& +\frac{1}{12} R\left(H_{\dot{a} I \dot{c} K} H^{\dot{c} K \dot{a} I}+\Sigma_{\dot{a} I}^{c K} \Sigma_{c K}^{\dot{a} I}+H_{a I}^{\dot{c} K} H_{\dot{c} K}^{a I}\right)+\frac{1}{2}\left|H_{\dot{a} I \dot{c} K} H^{\dot{c} K \dot{b} J}\right|^{2} \\
& \left.+2 H_{\dot{a} I \dot{c} K} \Sigma_{b J}^{\dot{c} K} H^{\dot{a} I \dot{d} L} \Sigma_{d L}^{b J}+\Sigma_{a I}^{\dot{c} K} \Sigma_{\dot{c} K}^{b J} \Sigma_{b J}^{\dot{d L}} \sum_{\dot{d} L}^{a I}\right]
\end{aligned}
$$

Using the identities

$$
\begin{aligned}
R_{\mu \nu \rho \sigma}^{2} & =2 C_{\mu \nu \rho \sigma}^{2}+\frac{1}{3} R^{2}-R^{*} R^{*} \\
R_{\mu \nu}^{2} & =\frac{1}{2} C_{\mu \nu \rho \sigma}^{2}+\frac{1}{3} R^{2}-\frac{1}{2} R^{*} R^{*}
\end{aligned}
$$

where $R^{*} R^{*}=\frac{1}{4} \epsilon^{\mu \nu \rho \sigma} \epsilon_{\alpha \beta \gamma \delta} R_{\mu \nu}^{\alpha \beta} R_{\rho \sigma}^{\gamma \delta}$.

$$
\begin{aligned}
\frac{1}{30}\left(5 R^{2}-8 R_{\mu \nu}^{2}-7 R_{\mu \nu \rho \sigma}^{2}\right) & =R^{2} \frac{1}{30}\left(5-\frac{8}{3}-\frac{7}{3}\right)+\frac{1}{30} C_{\mu \nu \rho \sigma}^{2}(-4-14)+\frac{1}{30} R^{*} R^{*}(4+7) \\
& =-\frac{3}{5} C_{\mu \nu \rho \sigma}^{2}+\frac{11}{30} R^{*} R^{*}
\end{aligned}
$$

Then $a_{4}$ simplifies to

$$
\begin{aligned}
a_{4}=\frac{1}{2 \pi^{2}} \int & d^{4} x \sqrt{g}\left[-\frac{3}{5} C_{\mu \nu \rho \sigma}^{2}+\frac{11}{30} R^{*} R^{*}+g_{L}^{2}\left(W_{\mu \nu L}^{\alpha}\right)^{2}+g_{R}^{2}\left(W_{\mu \nu R}^{\alpha}\right)^{2}+g^{2}\left(V_{\mu \nu}^{m}\right)^{2}\right. \\
& +\nabla_{\mu} \Sigma_{a I}^{\dot{c} K} \nabla^{\mu} \Sigma_{\dot{c} K}^{a I}+\frac{1}{2} \nabla_{\mu} H_{\dot{a} I \dot{b} J} \nabla^{\mu} H^{\dot{a} I \dot{b} J}+\frac{1}{12} R\left(H_{\dot{a} I \dot{c} K} H^{\dot{c} K \dot{a} I}+2 \Sigma_{\dot{a} I}^{c K} \Sigma_{c K}^{\dot{a} I}\right) \\
& \left.+\frac{1}{2}\left|H_{\dot{a} I \dot{c} K} H^{\dot{c} K \dot{b} J}\right|^{2}+2 H_{\dot{a} I \dot{c} K} \Sigma_{b J}^{\dot{c} K} H^{\dot{a} I \dot{d} L} \sum_{\dot{d} L}^{b J}+\Sigma_{a I}^{\dot{c} K} \sum_{\dot{c} K}^{b J} \Sigma_{b J}^{\dot{d} L} \Sigma_{\dot{d} L}^{a I}\right]
\end{aligned}
$$

\section{Acknowledgments}

AHC is supported in part by the National Science Foundation under Grant No. Phys0854779 and Phys-1202671. WDvS thanks IHÉS for hospitality during a visit from JanuaryMarch 2013.

Open Access. This article is distributed under the terms of the Creative Commons Attribution License which permits any use, distribution and reproduction in any medium, provided the original author(s) and source are credited. 


\section{References}

[1] K. Babu and R. Mohapatra, Coupling unification, GUT scale baryogenesis and neutron-antineutron oscillation in SO(10), Phys. Lett. B 715 (2012) 328 [arXiv:1206.5701] [INSPIRE].

[2] J.W. Barrett A lorentzian version of the noncommutative geometry of the standard model of particle physics, J. Math. Phys. 48 (2007) 012303 [hep-th/0608221] [INSPIRE].

[3] A. Connes, Gravity coupled with matter and the foundation of non-commutative geometry, Commun. Math. Phys. 182 (1996) 155 [hep-th/9603053] [INSPIRE].

[4] A. Connes, Noncommutative geometry and the standard model with neutrino mixing, JHEP 11 (2006) 081 [hep-th/0608226] [INSPIRE].

[5] A.H. Chamseddine and A. Connes, Universal formula for noncommutative geometry actions: unification of gravity and the standard model, Phys. Rev. Lett. 77 (1996) 4868 [INSPIRE].

[6] A.H. Chamseddine and A. Connes, The spectral action principle, Commun. Math. Phys. 186 (1997) 731 [hep-th/9606001] [INSPIRE].

[7] A.H. Chamseddine and A. Connes, Inner fluctuations of the spectral action, J. Geom. Phys. 57 (2006) 1 [hep-th/0605011] [INSPIRE].

[8] A.H. Chamseddine and A. Connes, Conceptual explanation for the algebra in the noncommutative approach to the standard model, Phys. Rev. Lett. 99 (2007) 191601 [arXiv:0706.3690] [INSPIRE].

[9] A.H. Chamseddine and A. Connes, Why the standard model, J. Geom. Phys. 58 (2008) 38 [arXiv:0706.3688] [INSPIRE].

[10] A.H. Chamseddine and A. Connes, Noncommutative geometry as a framework for unification of all fundamental interactions including gravity. Part I, Fortschr. Phys. 58 (2010) 553 [arXiv: 1004.0464] [INSPIRE].

[11] A.H. Chamseddine and A. Connes, Resilience of the spectral standard model, JHEP 09 (2012) 104 [arXiv: 1208.1030] [InSPIRE].

[12] A.H. Chamseddine, A. Connes and M. Marcolli, Gravity and the standard model with neutrino mixing, Adv. Theor. Math. Phys. 11 (2007) 991 [hep-th/0610241] [InSPIRE].

[13] A.H. Chamseddine, A. Connes and W.D. van Suijlekom, Inner fluctuations in noncommutative geometry without the first order condition, J. Geom. Phys. 73 (2013) 222 [arXiv:1304.7583] [INSPIRE].

[14] L. Dạbrowski, F. D'Andrea, G. Landi and E. Wagner, Dirac operators on all Podles quantum spheres, J. Noncomm. Geom. 1 (2007) 213 [math/0606480].

[15] L. Dąbrowski, G. Landi, M. Paschke and A. Sitarz, The spectral geometry of the equatorial Podles sphere, C. R. Acad. Sci. Paris Ser. I 340 (2005) 819.

[16] L. Dạbrowski, G. Landi, A. Sitarz, W.D. van Suijlekom and J.C. Várilly, The Dirac operator on $\mathrm{SU}_{q}(2)$, Commun. Math. Phys. 259 (2005) 729.

[17] L. Da̧browski, G. Landi, A. Sitarz, W.D. van Suijlekom and J.C. Várilly, Local index formula for $\mathrm{SU}_{q}(2)$, K-Theor. 35 (2005) 375.

[18] A. Devastato, F. Lizzi and P. Martinetti, Grand symmetry, spectral action and the Higgs mass, arXiv:1304.0415 [INSPIRE]. 
[19] J. Elias-Miro, J.R. Espinosa, G.F. Giudice, H.M. Lee and A. Strumia, Stabilization of the electroweak vacuum by a scalar threshold effect, JHEP 06 (2012) 031 [arXiv:1203.0237] [INSPIRE].

[20] R.N. Mohapatra and R. Marshak, Local B-L symmetry of electroweak interactions, Majorana neutrinos and neutron oscillations, Phys. Rev. Lett. 44 (1980) 1316 [Erratum ibid. 44 (1980) 1643] [INSPIRE].

[21] J. Pati and A. Salam, Lepton number as the fourth color, Phys. Rev. D 10 (1974) 174. 